\title{
Critical nonlinear elliptic equations with singularities and cylindrical symmetry
}

\section{Marino Badiale and Enrico Serra}

\begin{abstract}
Motivated by a problem arising in astrophysics we study a nonlinear elliptic equation in $\mathbb{R}^{N}$ with cylindrical symmetry and with singularities on a whole subspace of $\mathbb{R}^{N}$. We study the problem in a variational framework and, as the nonlinearity also displays a critical behavior, we use some suitable version of the ConcentrationCompactness Principle. We obtain several results on existence and nonexistence of solutions.
\end{abstract}

\section{Introduction}

This paper continues the work begun in [1], which was concerned with the problem

$$
\left\{\begin{array}{l}
-\Delta u(x)=\phi(r)|u|^{p-2} u \text { in } \mathbb{R}^{3} \\
u(x)>0 \text { in } \mathbb{R}^{3} \\
u \in \mathcal{D}^{1,2}\left(\mathbb{R}^{3}\right) .
\end{array}\right.
$$

Here $x=\left(x_{1}, x_{2}, x_{3}\right) \in \mathbb{R}^{3}, r=\sqrt{x_{1}^{2}+x_{2}^{2}}$ and the model function for $\phi$ is

$$
\phi(r)=\frac{r^{2 \alpha}}{\left(1+r^{2}\right)^{\alpha+\frac{1}{2}}},
$$

where $\alpha$ is a positive number.

The equation in (1.1) displays a cylindrical symmetry and its motivation comes from some research in astrophysics where it was proposed as a model for the study of elliptic galaxies (see [9]).

2000 Mathematics Subject Classification: critical exponents, loss of compactness, cylindrical symmetry, singularities.

Keywords: 35J20, 35B33. 
There is a vast literature concerning the modeling of astronomical objects like galaxies or globular clusters of stars (we refer to the bibliography in [1]) and several elliptic equations arising from this questions have been investigated. As far as we know most of these models are radial, because they model radial objects (e.g. globular clusters of stars). Much less is known in the case of cylindrical symmetry (derived from the axial symmetry of elliptic galaxies) and many interesting problems from the mathematical point of view are still open.

An analysis of the model proposed in [9] was carried out in the paper [1], where it was proved that problem (1.1) can be handled by a variational approach in the case $p \in[4,6]$, and where various results about existence and nonexistence of solutions were obtained.

The case $p=4$ turned out to be particularly interesting since for the problem

$$
\left\{\begin{array}{l}
-\Delta u(x)=\phi(r) u^{3} \quad \text { in } \quad \mathbb{R}^{3} \\
u(x)>0 \text { in } \quad \mathbb{R}^{3} \\
u \in \mathcal{D}^{1,2}\left(\mathbb{R}^{3}\right)
\end{array}\right.
$$

only a negative result was obtained, that is, the nonexistence of solutions for the minimization problem associated to (1.3) when $\phi(r)$ satisfies suitable hypotheses, and in particular when it has exactly the form (1.2). The reason for this is that in the case $p=4$ the equation has a critical nature. Indeed, as $\phi(r)$ is asymptotic to $1 / r$ when $r \rightarrow \infty$, problem (1.3) admits the equation

$$
-\Delta u(x)=\frac{1}{r} u^{3} \quad \text { in } \quad \mathbb{R}^{3}
$$

as a "limiting" problem, and this is critical in the usual sense, namely it is invariant with respect to a class of dilations. If $u$ is a solution of (1.4), then $u_{\lambda}(x)=\lambda^{1 / 2} u(\lambda x)$ is still a solution. As usual, this invariance generates some lack of compactness for the Palais-Smale sequences when dealing with the variational problem associated to (1.4), and this in turn takes place also in problem (1.3), in the form of an invariance manifested by the limiting equation.

Motivated by these observations, in this paper we study equation (1.3) and a more general problem in $\mathbb{R}^{N}$, with $N \geq 3$, given by

$$
\left\{\begin{array}{l}
-\Delta u(x)=\phi\left(\left|x^{\prime}\right|\right) u^{2_{*}-1} \quad \text { in } \mathbb{R}^{N} \\
u(x)>0 \text { in } \mathbb{R}^{N} \\
u \in \mathcal{D}^{1,2}\left(\mathbb{R}^{N}\right)
\end{array}\right.
$$

where we have set $x=\left(x^{\prime}, z\right) \in \mathbb{R}^{K} \times \mathbb{R}^{N-K}(2 \leq K \leq N)$ and $2_{*}=2 \frac{N-s}{N-2}$, with $0<s<2$; the function $\phi(r)$ is asymptotic, at 0 or at $\infty$ (or both), 
to the function $1 / r^{s}$. Exactly as problem (1.3) admits (1.4) as a limiting equation, the more general problem (1.5) admits

$$
\left\{\begin{array}{l}
-\Delta u(x)=\frac{1}{\left|x^{\prime}\right|^{s}} u^{2 *-1} \quad \text { in } \quad \mathbb{R}^{N} \\
u(x)>0 \text { in } \mathbb{R}^{N} \\
u \in \mathcal{D}^{1,2}\left(\mathbb{R}^{N}\right)
\end{array}\right.
$$

as a limiting problem, and this is critical because it is invariant with respect to the scalings

$$
u_{\lambda}(x)=\lambda^{\frac{N-2}{2}} u(\lambda x) .
$$

We will associate to (1.3) or to (1.5) a variational formulation and we will give some sufficient conditions for their solvability. As usual in critical problems, these conditions are expressed as strict inequalities between critical levels (see Theorem 3.1). For this abstract result we use a nice version of the celebrated Concentration-Compactness principles of P. L. Lions. This version, which in some sense gives a unified treatment of the different ConcentrationCompactness principles, is due to S. Solimini [16]. We then give some easy applications of the existence result, and we also describe some cases where nonexistence occurs (see theorems 4.1 and 4.2).

In the particular case of problem (1.3) we obtain some additional results. To begin with, we obtain some information about regularity and decay properties of solutions (Lemmas 2.2 and Corollary 4.6), and from these results we obtain the existence of solutions in the case in which the $\phi$ 's are small perturbations of the function (1.2), for which, as proved in [1], the minimization problem associated to (1.3) has no solutions. These results show, once again, how critical problems are sensitive to perturbations.

Critical problems with nonlinearities exhibiting a singularity have been much studied in recent years (see for example $[5,6,7,8,10,12,18]$ ), but to our knowledge in the past literature the singularity is always concentrated at a point, while in our case there is a whole subspace of singularities. The only exception that we are aware of is the paper [14] by $\mathrm{Ni}$, that studies an equation of type (1.5) where $K \geq 3$ and where the existence of solutions bounded away from zero is established. These results are therefore very different in spirit from ours.

For problems with singularities and cylindrical symmetry much work has still to be done, for example about regularity or uniqueness of solutions, even for the limiting equation in (1.6). Note for instance that this equation cannot be reduced to an ordinary differential equation as is the case for example with the analysis of the minimizers of the usual Sobolev quotients; therefore problems of uniqueness of positive solutions appear to be genuinely multi-dimensional and a general approach is still lacking. 
The present paper, along with [1], presents some initial results.

In Section 2 we give some preliminary results, we describe the regularity properties and we give some nonexistence results. Section 3 is devoted to the proof of the main compactness result, which is then applied in Section 4 to prove existence of solutions for problems (1.3) and (1.5).

After this work was completed, we became aware of the paper [15] where some results similar to ours are obtained as far as existence problems are concerned. Our results however treat also regularity, Pohozaev identities and decay properties, and we make an analysis of the lack of compactness for the minimizing sequences associated to problem (1.5).

\section{Notation}

We collect below a list of the main notation used throughout the paper.

- For any $x=\left(x_{1} \ldots, x_{N}\right) \in \mathbb{R}^{N}$ and $K \geq 2$ we write $x=\left(x^{\prime}, z\right)$ with $x^{\prime}=\left(x_{1}, \ldots, x_{K}\right) \in \mathbb{R}^{K}$ and $z=\left(x_{K+1}, \ldots, x_{N}\right) \in \mathbb{R}^{N-K}$. We also write $r$ for $\left|x^{\prime}\right|$. We define $A=\left\{x \in \mathbb{R}^{N} \mid x^{\prime} \neq 0\right\}$.

- $\mathcal{D}^{1,2}\left(\mathbb{R}^{N}\right)$ is the closure of $C_{0}^{\infty}\left(\mathbb{R}^{N}\right)$ with respect to the norm

$$
\|u\|=\left(\int_{\mathbb{R}^{N}}|\nabla u|^{2} d x\right)^{1 / 2} .
$$

As no misunderstanding is possible, we will also write $\mathcal{D}^{1,2}$ instead of $\mathcal{D}^{1,2}\left(\mathbb{R}^{N}\right)$.

- $L^{q}=L^{q}\left(\mathbb{R}^{N}\right), L_{l o c}^{q}=L_{l o c}^{q}\left(\mathbb{R}^{N}\right)$ are the usual Lebesgue spaces. For a Lebesgue-measurable function $g$ we will write $L^{q}(g)$ to mean the space $L^{q}\left(\mathbb{R}^{N}\right)$ with respect to the measure $g(x) d x$.

- $2^{*}=\frac{2 N}{N-2}$ is the critical exponent for the Sobolev embedding and $2_{*}=2_{*}(s)$ is $\frac{2(N-s)}{N-2}$.

- For any $P \in \mathbb{R}^{N}$ and $r>0$ we denote by $B(P, r)$ or $B_{r}(P)$ the open ball in $\mathbb{R}^{N}$ with center $P$ and radius $r$, while $B_{r}=B(0, r)$.

- We will use $C$ to denote any positive constant, that can change from line to line.

- $x \cdot y$ is the usual scalar product in $\mathbb{R}^{N}$.

- We set $\mathbb{R}^{+}=\left[0,+\infty\left[\right.\right.$ and $\left.\mathbb{R}_{+}=\right] 0,+\infty[$. 


\section{Preliminary results}

We first give our main hypotheses on the function $\phi$. We assume that for some $\eta \in(0,1)$ and $s \in(0,2)$

$$
\phi \in C_{l o c}^{0, \eta}\left(\mathbb{R}_{+}, \mathbb{R}^{+}\right), \quad \phi(r) r^{s} \in L^{\infty}\left(\mathbb{R}_{+}\right),
$$

(2.1) and at least one between $\lim _{r \rightarrow 0} \phi(r) r^{s}=1$ and $\lim _{r \rightarrow+\infty} \phi(r) r^{s}=1$ holds.

As we said in the introduction, we will obtain existence results for problem (1.5) by the study of a minimization problem of a standard type. The basic tool here is an embedding result obtained in [1], reminiscent of the inequalities in [4], which we recall in a form well-suited for our study. In its statement we consider $\mathbb{R}^{N}=\mathbb{R}^{K} \times \mathbb{R}^{N-K}$, with $K \geq 2$, and we set $x^{\prime}=\left(x_{1}, \ldots, x_{K}\right)$.

Theorem 2.1 (Theorem 2.1 in [1]) For each $0<s<2$, let $2_{*}=\frac{2(N-s)}{N-2}$. Then there exists a positive constant $C_{s}$ such that, for all $u \in \mathcal{D}^{1,2}\left(\mathbb{R}^{N}\right)$,

$$
\int_{\mathbb{R}^{N}} \frac{1}{\left|x^{\prime}\right|^{s}}|u|^{2 *} d x \leq C_{s}\left(\int_{\mathbb{R}^{N}}|\nabla u|^{2} d x\right)^{2 * / 2}
$$

Theorem 2.1 implies that in the space $\mathcal{D}^{1,2}\left(\mathbb{R}^{N}\right)$ the integral $\int_{\mathbb{R}^{N}} \frac{1}{\left|x^{\prime}\right|^{s}}|u|^{2 *} d x$ is finite, so, for $u \in \mathcal{D}^{1,2}\left(\mathbb{R}^{N}\right) \backslash\{0\}$, we can define

$$
J(u)=\frac{\int_{\mathbb{R}^{N}}|\nabla u|^{2} d x}{\left(\int_{\mathbb{R}^{N}} \frac{1}{\left|x^{\prime}\right|^{s}}|u|^{2_{*}} d x\right)^{2 / 2_{*}}}, \quad \text { and } \quad J_{\phi}(u)=\frac{\int_{\mathbb{R}^{N}}|\nabla u|^{2} d x}{\left(\int_{\mathbb{R}^{N}} \phi\left(\left|x^{\prime}\right|\right)|u|^{2 *} d x\right)^{2 / 2_{*}}} .
$$

It is easy to see that $J$ and $J_{\phi}$ are $C^{1}$ functionals over $\mathcal{D}^{1,2}$. We also define

$$
S=\inf \left\{J(u) \mid u \in \mathcal{D}^{1,2} \backslash\{0\}\right\} \quad \text { and } \quad S_{\phi}=\inf \left\{J_{\phi}(u) \mid u \in \mathcal{D}^{1,2} \backslash\{0\}\right\},
$$

and we consider the minimization problem

$$
\text { find } u \in \mathcal{D}^{1,2} \backslash\{0\}, \quad u \geq 0 \text {, such that } J_{\phi}(u)=S_{\phi} .
$$

A solution of (2.3) gives, up to a constant, a good candidate to solve problem (1.5). Indeed, usual arguments imply that a solution of (2.3), suitably normalized, yields a weak solution to the equation in (1.5). Due to the presence of $\phi$ this solution may be singular on $x^{\prime}=0$, and therefore the condition $u>0$ in $\mathbb{R}^{N}$ must be interpreted with some care. In view of further applications we now investigate the regularity and positivity of solutions in some particular cases.

By standard elliptic regularity theory, it is obvious that the solutions of (1.5) are $C^{2}$ when $x^{\prime} \neq 0$. The regularity across $x^{\prime}=0$ depends on the values of $s$ and $N$. 
Lemma 2.2 Assume (2.1) and let $u \in \mathcal{D}^{1,2}, u \geq 0$, be a weak solution of the equation

$$
-\Delta u(x)=\phi\left(\left|x^{\prime}\right|\right) u^{2 *-1}
$$

If $s N<4$, then $u \in C_{\text {loc }}^{0, \theta}\left(\mathbb{R}^{N}\right)$ for some $\theta \in(0,1)$.

If $s N<2$, then $u \in C_{\text {loc }}^{1, \theta}\left(\mathbb{R}^{N}\right)$ for some $\theta \in(0,1)$.

Proof. We first claim that $u \in L_{\text {loc }}^{q}\left(\mathbb{R}^{N}\right)$ for all $q<+\infty$. To prove this we use a result due to Brézis and Kato [2], that we apply in the form that one finds in the book of Struwe (Lemma B.3 p. 218 in [17]).

We write

$$
\phi\left(\left|x^{\prime}\right|\right) u^{2_{*}-1}=\phi\left(\left|x^{\prime}\right|\right) \frac{u^{2_{*}-1}}{1+u}(1+u),
$$

and we set

$$
a(x)=\phi\left(\left|x^{\prime}\right|\right) \frac{u^{2 *-1}(x)}{1+u(x)},
$$

so that $u$ satisfies

$$
-\Delta u=a(x)(1+u) .
$$

We now prove that $a \in L^{N / 2}\left(\mathbb{R}^{N}\right)$. Once this is proved, Lemma B.3 in [17] implies our claim, that is, $u \in L_{l o c}^{q}\left(\mathbb{R}^{N}\right)$ for all $q<+\infty$. To prove that $a \in L^{N / 2}\left(\mathbb{R}^{N}\right)$, we write

$$
a(x)=\phi\left(\left|x^{\prime}\right|\right)\left|x^{\prime}\right|^{s} \frac{u^{2 *-2}}{\left|x^{\prime}\right|^{s}} \frac{u}{1+u}
$$

and notice that, of course, $\frac{u}{1+u}$ is bounded (as $u \geq 0$ ), while $\phi\left(\left|x^{\prime}\right|\right)\left|x^{\prime}\right|^{s}$ is bounded by (2.1). Hence we have to prove that

$$
\frac{u^{2 *-2}}{\left|x^{\prime}\right|^{s}} \in L^{N / 2}, \quad \text { that is, } \quad \frac{u^{\left(2_{*}-2\right) N / 2}}{\left|x^{\prime}\right|^{N s / 2}} \in L^{1} .
$$

To show this we notice that since $s N<4$, so that $N s / 2<2<K$, we can use the embedding results of [1] in the form (2.2). We just have to realize that the exponents match correctly, namely

$$
\left(2_{*}-2\right) \frac{N}{2}=\frac{2(N-N s / 2)}{N-2},
$$

as a trivial computation shows. In this way we have proved the claim.

To complete the proof we observe that since $u \in L_{l o c}^{q}$ for all $q<\infty$, the local integrability of $\left(\frac{u^{2 *-1}}{\left|x^{\prime}\right|^{s}}\right)^{p}$ can be deduced from the local integrability of $\left(\frac{1}{\left|x^{\prime}\right|^{s}}\right)^{p}$. 
Now if $s N<4$ we can take some $p>\frac{N}{2}$ such that $s p<2$; since $K \geq 2$, we see that $\left(\frac{1}{\left|x^{\prime}\right|}\right)^{s p}$ is locally integrable. This implies that $\frac{u^{2 *-1}}{\left|x^{\prime}\right|^{s}}$ is locally in $L^{q}$ for some $p>q>\frac{N}{2}$. By the usual elliptic regularity theory and the Sobolev embeddings, it follows that $u \in C_{\text {loc }}^{0, \theta}\left(\mathbb{R}^{N}\right)$ for some $\theta \in(0,1)$.

Finally, if $s N<2$ we can repeat the above argument using some $p>N$ such that again $s p<2$, to obtain that $u \in C_{l o c}^{1, \theta}\left(\mathbb{R}^{N}\right)$ for some $\theta \in(0,1)$.

Remark 2.3 Regularity questions for general $N$ and $s$ are open, but we notice that not much more that the previous results is to be expected. Indeed, equation (1.4) when $K=N=3$ admits the solution $u(x)=\sqrt{2}(1+|x|)^{-1}$, which is only Lipschitz continuous. Singular solutions might also be present in some cases.

Notice however that the previous lemma covers some important particular cases such as problem (1.3). Furthermore, we remark that in the astrophysical problem the function $\phi$ is not singular at zero (see (1.2)), so that in this case the solutions are classical.

We now turn to the question of the positivity of solutions in all $\mathbb{R}^{N}$.

Lemma 2.4 Assume (2.1) and let $u \in \mathcal{D}^{1,2}, u \geq 0$, be a weak solution of the equation

$$
-\Delta u(x)=\phi\left(\left|x^{\prime}\right|\right) u^{2_{*}-1} .
$$

If $s N<4$, then $u>0$ in $\mathbb{R}^{N}$.

Proof. By standard elliptic regularity, $u \in C_{l o c}^{2, \eta}(A)$ for some $\eta \in(0,1)$, where we recall that $A=\left\{x \in \mathbb{R}^{N} \mid x^{\prime} \neq 0\right\}$; hence we can apply the classical strong maximum principle to obtain that $u>0$ in $A$.

To conclude we first notice that, by Lemma $2.2, u \in C_{l o c}^{0, \theta}$, for some $\theta \in$ $(0,1)$. Let $x_{0} \in \mathbb{R}^{N}$ be such that $x_{0}^{\prime}=0$ and consider the ball $B=B_{1}\left(x_{0}\right)$. Define $\tilde{\phi}(r)=\min \{1, \phi(r)\}$ and remark that $\tilde{\phi}$ is Hölder continuous, because so is $\phi$. Let $v$ be the classical solution of the problem

$$
\left\{\begin{array}{l}
-\Delta v(x)=\tilde{\phi}\left(\left|x^{\prime}\right|\right) u^{2 *-1} \quad \text { in } B \\
v(x)=0 \text { on } \partial B .
\end{array}\right.
$$

Notice that $\tilde{\phi}\left(\left|x^{\prime}\right|\right) u^{2 *-1}$ is Hölder continuous. Clearly now $-\Delta u \geq-\Delta v$ in the weak sense in $B$, and $u \geq v$ on $\partial B$, so that, using the maximum principle for weak solutions, we have $u \geq v$ in all of $B$. But $v$ is a classical solution of (2.4), $-\Delta v \geq 0$ and $v$ is not a constant, so by the strong classical maximum principle we have $v>0$ in all of $B$ and hence also $u\left(x_{0}\right)>0$. 
We now want to prove some Pohozaev-type result for (1.5). We will obtain that, under some very natural hypotheses, (1.5) has no solutions. In the next section we also will prove some existence results for (1.5) and, as usual in critical problems, we will see that one passes from existence to non existence of solutions by small perturbations.

The first nonexistence result is a consequence of the following identity of Pohozaev type. The validity of this identity is affected by the regularity properties of the solutions, and for this reason we will state it in a form that will suit our applications.

The argument to derive the identity is the standard one (see for example the book of Kuzin and Pohozaev [13], Theorem 29.4), except of course for the fact that in general our solutions are not $C^{2}$ in all of $\mathbb{R}^{N}$.

Proposition 2.5 Let $a: \mathbb{R}^{N} \rightarrow \mathbb{R}$ be a function such that

$$
a(x)\left|x^{\prime}\right|^{s} \in L^{\infty}\left(\mathbb{R}^{N}\right), \quad \text { with } \quad a \in C(A),
$$

and let $u \in \mathcal{D}^{1,2}\left(\mathbb{R}^{N}\right)$ be a weak solution of

$$
-\Delta u=a(x)|u|^{p-2} u \quad \text { in } \quad \mathbb{R}^{N} .
$$

Assume that $u \in C_{\text {loc }}^{1, \theta}\left(\mathbb{R}^{N}\right) \cap L^{p}\left(\mathbb{R}^{N}\right) \cap C^{2}(A)$ for some $\theta \in(0,1)$ and also that

$$
a|u|^{p} \in L^{1}\left(\mathbb{R}^{N}\right) \quad \text { and } \quad \nabla a(x) \cdot x|u|^{p} \in L^{1}\left(\mathbb{R}^{N}\right) .
$$

Then the following identity holds:

$$
\int_{\mathbb{R}^{N}}\left[\left(\frac{N-2}{2}-\frac{N}{p}\right) a(x)-\frac{1}{p} \nabla a(x) \cdot x\right]|u|^{p} d x=0 .
$$

Proof. For any $R, \epsilon>0$ we consider the ball $B_{R}$ and the sets

$$
\Omega_{1}=\Omega_{1}(R, \epsilon)=\left\{x \in B_{R} \mid x_{1}>\epsilon\right\}, \quad \Omega_{2}=\Omega_{2}(R, \epsilon)=\left\{x \in B_{R} \mid x_{1}<-\epsilon\right\} .
$$

By our assumptions, $u$ is a classical solution of (2.6) in $\Omega_{1} \cup \Omega_{2}$, so in each of these sets we can repeat the standard arguments one uses to derive the Pohozaev identity, that is, we multiply (2.6) by $x \cdot \nabla u(x)$ and we integrate. Recalling that

$$
x \cdot \nabla u \Delta u=\operatorname{div}\left((x \cdot \nabla u) \nabla u-\frac{1}{2}|\nabla u|^{2} x\right)+\frac{N-2}{2}|\nabla u|^{2}
$$

and

$$
x \cdot \nabla u a(x)|u|^{p-2} u=\operatorname{div}\left(\frac{1}{p} a(x)|u|^{p} x\right)-\frac{N}{p} a(x)|u|^{p}-\frac{1}{p}|u|^{p} \nabla a(x) \cdot x,
$$


we obtain, setting $\Omega=\Omega_{1} \cup \Omega_{2}$,

$$
\begin{aligned}
\int_{\partial \Omega} & \left(-x \cdot \nabla u \frac{\partial u}{\partial n}+\frac{1}{2}|\nabla u|^{2} x \cdot n\right) d \sigma-\frac{N-2}{2} \int_{\Omega}|\nabla u|^{2} d x \\
= & \int_{\partial \Omega} \frac{1}{p} a(x)|u|^{p} x \cdot n d \sigma-\frac{N}{p} \int_{\Omega} a(x)|u|^{p} d x-\frac{1}{p} \int_{\Omega} x \cdot \nabla a|u|^{p} d x,
\end{aligned}
$$

that is

$$
\begin{aligned}
\int_{\partial \Omega} & \left(-x \cdot \nabla u \frac{\partial u}{\partial n}+\frac{1}{2}|\nabla u|^{2} x \cdot n-\frac{1}{p} a(x)|u|^{p} x \cdot n\right) d \sigma \\
= & \frac{N-2}{2} \int_{\Omega}|\nabla u|^{2} d x-\frac{1}{p} \int_{\Omega}(N a(x)+\nabla a(x) \cdot x)|u|^{p} d x .
\end{aligned}
$$

Here $n$ is the outward normal at $\partial \Omega$ and $d \sigma$ is the $(N-1)$-dimensional measure of $\partial \Omega$. Now we pass to the limit in (2.9) as $\epsilon \rightarrow 0$. It is obvious that the limit of the right hand side in (2.9) is

$$
\frac{N-2}{2} \int_{B_{R}}|\nabla u|^{2} d x-\frac{1}{p} \int_{B_{R}}(N a(x)+\nabla a(x) \cdot x)|u|^{p} d x .
$$

For the left hand side, we have

$$
\begin{aligned}
\partial \Omega= & \left\{x \in \partial B_{R} \mid x_{1}>\epsilon\right\} \cup\left\{x \in \partial B_{R} \mid x_{1}<-\epsilon\right\} \cup\left\{x \in B_{R} \mid x_{1}=\epsilon\right\} \\
& \cup\left\{x \in B_{R} \mid x_{1}=-\epsilon\right\} \\
= & S_{1} \cup S_{2} \cup S_{3} \cup S_{4},
\end{aligned}
$$

with obvious definitions of $S_{i}=S_{i}(\epsilon)$. Again it is obvious that

$$
\begin{aligned}
\lim _{\epsilon \rightarrow 0} \int_{S_{1} \cup S_{2}} & \left(-x \cdot \nabla u \frac{\partial u}{\partial n}+\frac{1}{2}|\nabla u|^{2} x \cdot n-\frac{1}{p} a(x)|u|^{p} x \cdot n\right) d \sigma \\
= & \int_{\partial B_{R}}\left(-x \cdot \nabla u \frac{\partial u}{\partial n}+\frac{1}{2}|\nabla u|^{2} x \cdot n-\frac{1}{p} a(x)|u|^{p} x \cdot n\right) d \sigma .
\end{aligned}
$$

We have now to see what happens in $S_{3} \cup S_{4}$ as $\epsilon \rightarrow 0$. Notice that in $S_{3}$, $n=(-1,0, \ldots, 0)$, while in $S_{4}, n=(1,0, \ldots, 0)$. Hence we can write

$$
\begin{aligned}
\int_{S_{3} \cup S_{4}}( & \left.-x \cdot \nabla u \frac{\partial u}{\partial n}+\frac{1}{2}|\nabla u|^{2} x \cdot n-\frac{1}{p} a(x)|u|^{p} x \cdot n\right) d \sigma \\
= & \int_{S_{3}}\left(x \cdot \nabla u D_{1} u-\frac{1}{2}|\nabla u|^{2} x_{1}+\frac{1}{p} a(x)|u|^{p} x_{1}\right) d \sigma \\
& +\int_{S_{4}}\left(-x \cdot \nabla u D_{1} u+\frac{1}{2}|\nabla u|^{2} x_{1}-\frac{1}{p} a(x)|u|^{p} x_{1}\right) d \sigma .
\end{aligned}
$$


By the hypotheses, the function $-x \cdot \nabla u D_{1} u+\frac{1}{2}|\nabla u|^{2} x_{1}-\frac{1}{p} a(x)|u|^{p} x_{1}$ is continuous everywhere, so that we can conclude that

$$
\lim _{\epsilon \rightarrow 0} \int_{S_{3} \cup S_{4}}\left(-x \cdot \nabla u \frac{\partial u}{\partial n}+\frac{1}{2}|\nabla u|^{2} x \cdot n-\frac{1}{p} a(x)|u|^{p} x \cdot n\right) d \sigma=0 .
$$

Hence, after passing to the limit as $\epsilon \rightarrow 0,(2.9)$ implies

$$
\begin{aligned}
\int_{\partial B_{R}} & \left(-x \cdot \nabla u \frac{\partial u}{\partial n}+\frac{1}{2}|\nabla u|^{2} x \cdot n-\frac{1}{p} a(x)|u|^{p} x \cdot n\right) d \sigma= \\
& =\frac{N-2}{2} \int_{B_{R}}|\nabla u|^{2} d x-\frac{1}{p} \int_{B_{R}}(N a(x)+\nabla a(x) \cdot x)|u|^{p} d x .
\end{aligned}
$$

Now the argument can proceed in the standard way (see [13]), so we will be sketchy. The hypotheses $|\nabla u| \in L^{2}$, and $a|u|^{p} \in L^{1}$ imply that there is a sequence of radii $R_{n} \rightarrow+\infty$ such that

$$
\lim _{n \rightarrow+\infty} \int_{\partial B_{R_{n}}}\left(-x \cdot \nabla u \frac{\partial u}{\partial n}+\frac{1}{2}|\nabla u|^{2} x \cdot n-\frac{1}{p} a(x)|u|^{p} x \cdot n\right) d \sigma=0 .
$$

Therefore, passing to the limit in (2.10) we obtain

$$
\frac{N-2}{2} \int_{\mathbb{R}^{N}}|\nabla u|^{2} d x-\frac{1}{p} \int_{\mathbb{R}^{N}}(N a(x)+\nabla a(x) \cdot x)|u|^{p} d x=0 .
$$

But since $\int_{\mathbb{R}^{N}}|\nabla u|^{2} d x=\int_{\mathbb{R}^{N}} a|u|^{p} d x$, the result follows.

We now apply this Pohozaev identity to problem (1.5), with the suitable limitations on the values of $s$ and $N$.

Corollary 2.6 Assume that $s N<2$, that $\phi$ satisfies (2.1) and also that

$$
\phi^{\prime} \in C\left(\mathbb{R}_{+}\right) \quad \text { and } \quad \phi^{\prime}(r) r^{s+1} \in L^{\infty}\left(\mathbb{R}_{+}\right) .
$$

Define $\psi(r)=\phi(r) r^{s}$ and assume that $\psi$ is monotone (increasing or decreasing) and not constant. Then problem (1.5) has no solutions.

Proof. We apply Proposition 2.5 with $p=2_{*}$ and $a(x)=\phi\left(\left|x^{\prime}\right|\right)$. Let us notice that $\nabla a(x) \cdot x=\phi^{\prime}\left(\left|x^{\prime}\right|\right)\left|x^{\prime}\right|$, so that (2.1), (2.11) and Theorem 2.1 imply that the hypotheses (2.5) and (2.7) of Proposition 2.5 are satisfied. So, if we assume that $u$ is a solution of (1.5), by Lemma 2.2 we have $u \in C_{l o c}^{1, \theta}$ and by Lemma 2.4 we obtain that $u>0$ everywhere. 
Moreover, computing

$$
\begin{aligned}
\left(\frac{N-2}{2}-\right. & \left.\frac{N}{2_{*}}\right) a(x)-\frac{1}{2_{*}} \nabla a(x) \cdot x=-\frac{s}{2_{*}} a(x)-\frac{1}{2_{*}} \nabla a(x) \cdot x \\
& =-\frac{1}{2_{*}}\left(s \phi\left(\left|x^{\prime}\right|\right)+\phi^{\prime}\left(\left|x^{\prime}\right|\right)\left|x^{\prime}\right|\right) \\
& =-\frac{1}{2_{*}} \frac{1}{\left|x^{\prime}\right|^{s-1}}\left(s\left|x^{\prime}\right|^{s-1} \phi\left(\left|x^{\prime}\right|\right)+\phi^{\prime}\left(\left|x^{\prime}\right|\right)\left|x^{\prime}\right|^{s}\right)=\frac{1}{2_{*}\left|x^{\prime}\right|^{s-1}} \psi^{\prime}\left(\left|x^{\prime}\right|\right),
\end{aligned}
$$

we see that (2.8) gives

$$
0=\int_{\mathbb{R}^{N}} \frac{1}{\left|x^{\prime}\right|^{s-1}} \psi^{\prime}\left(\left|x^{\prime}\right|\right)|u|^{2 *} d x
$$

Under the hypothesis that $\psi$ is monotone and not constant we obtain that $\psi^{\prime}$ is not zero and has constant sign. Therefore (2.12) gives a contradiction and this implies that (1.5) has no solutions.

Remark 2.7 Notice that the nonexistence result can be applied to problem (1.1), since its solutions are regular. Therefore we obtain a generalization of the nonexistence result in [1], which concerned only the solutions of the minimization problem associated to (1.1).

Let us consider now the minimization problem (2.3). We will prove that it has no solutions assuming some hypotheses different than those of Corollary 2.6. We first prove an inequality.

Lemma 2.8 Assume that $\phi$ satisfies (2.1). Then

$$
S_{\phi} \leq S
$$

Proof. We know that $S$ is achieved at some $w \in \mathcal{D}^{1,2}\left(\mathbb{R}^{N}\right)$ (Theorem 2.5 in [1]). Let us define, for $\lambda>0, w_{\lambda}(x)=\lambda^{\frac{N-2}{2}} w(\lambda x)$. Then, by a standard change of variables, $J\left(w_{\lambda}\right)=J(w)=S$, while

$$
J_{\phi}\left(w_{\lambda}\right)=\frac{\int_{\mathbb{R}^{N}}|\nabla w|^{2} d x}{\left(\int_{\mathbb{R}^{N}} \phi\left(\frac{\left|x^{\prime}\right|}{\lambda}\right) \frac{\left|x^{\prime}\right|^{s}}{\lambda^{s}} \frac{|w|^{2 *}}{\left|x^{\prime}\right|^{s}} d x\right)^{2 / 2_{*}}} .
$$

To fix ideas, assume that $\lim _{r \rightarrow+\infty} \phi(r) r^{s}=1$. Since $\frac{|w|^{2} *}{\left|x^{\prime}\right|^{s}} \in L^{1}\left(\mathbb{R}^{N}\right)$, it is easy to see that $J_{\phi}\left(w_{\lambda}\right) \rightarrow J(w)=S$ as $\lambda \rightarrow 0$. On the other hand, we have by definition $S_{\phi} \leq J_{\phi}\left(w_{\lambda}\right)$ for all $\lambda>0$, so that the conclusion easily follows. If $\lim _{r \rightarrow 0} \phi(r) r^{s}=1$, the argument is the same, letting $\lambda \rightarrow+\infty$. 
Theorem 2.9 Assume that $\phi$ satisfies (2.1) and also that

$$
\phi(r) r^{s} \leq 1 \forall r>0 \text { and } \phi\left(r_{0}\right) r_{0}^{s}<1 \text { for some } r_{0}>0 \text {. }
$$

Then problem (2.3) has no positive solutions.

Proof. Assume by contradiction that $u$ is a positive solution of (2.3). Hence $J_{\phi}(u)=S_{\phi}$ and $u(x)>0$. We then obtain

$$
\int_{\mathbb{R}^{N}} \phi\left(\left|x^{\prime}\right|\right) u^{2^{*}} d x<\int_{\mathbb{R}^{N}} \frac{1}{\left|x^{\prime}\right|^{s}} u^{2^{*}} d x
$$

which implies

$$
S_{\phi}=\frac{\int_{\mathbb{R}^{N}}|\nabla u|^{2} d x}{\left(\int_{\mathbb{R}^{N}} \phi\left(\left|x^{\prime}\right|\right) u^{2 *} d x\right)^{2 / 2_{*}}}>\frac{\int_{\mathbb{R}^{N}}|\nabla u|^{2} d x}{\left(\int_{\mathbb{R}^{N}} \frac{1}{\left|x^{\prime}\right|^{s}} u^{2_{*}} d x\right)^{2 / 2_{*}}} \geq S,
$$

contradicting Lemma 2.8 .

Remark 2.10 Corollary 2.6 and Theorem 2.9 suggest the following open question: suppose that $\phi(r) r^{s} \leq 1, \phi(r) r^{s} \neq 1$, but $\phi(r) r^{s}$ is not monotone. Then, by Theorem 2.9, the minimization problem (2.3) has no solutions, but Corollary 2.6 does not apply. Is there any solution of problem (1.5)?

\section{A compactness result}

Throughout this section we will use the following hypothesis on $\phi$ :

$$
\limsup _{r \rightarrow 0,+\infty} \phi(r) r^{s} \leq 1
$$

This section is devoted to the proof of the following Theorem 3.1. In the next section we will apply it to find some existence results for problem (1.5).

Theorem 3.1 Assume that $\phi$ satisfies (2.1) and (3.1). If $S_{\phi}<S$, then problem (1.5) has a solution.

The main tool we use to prove Theorem 3.1 is the following Theorem 3.3, due to Solimini, [16]. It is a version of the Concentration-Compactness Principle. To state Theorem 3.3 we first define rescalings of functions in $\mathcal{D}^{1,2}$.

Definition 3.2 For any fixed $\lambda>0, p \in \mathbb{R}^{N}$, the rescaling $T=T(\lambda, p)$ is the function

$$
T: \mathcal{D}^{1,2}\left(\mathbb{R}^{N}\right) \rightarrow \mathcal{D}^{1,2}\left(\mathbb{R}^{N}\right) \quad \text { defined by } \quad T v(x)=\lambda^{\frac{N-2}{2}} v(\lambda(x-p)) .
$$


Notice that if $T=T(\lambda, p)$ then $T^{-1}=T(1 / \lambda,-\lambda p)$, that is,

$$
T^{-1} v(x)=\lambda^{-\frac{N-2}{2}} v\left(\frac{1}{\lambda} x+p\right)
$$

Theorem 3.3 ([16]) Assume that $\left\{v_{k}\right\}_{k}$ is a bounded sequence in $\mathcal{D}^{1,2}\left(\mathbb{R}^{N}\right)$. Then, up to a subsequence, one of the following alternatives holds:

(i) $v_{k} \rightarrow 0$ strongly in $L^{2^{*}}\left(\mathbb{R}^{N}\right)$.

(ii) There is a sequence $\left\{T_{k}\right\}_{k}$ of rescalings such that $T_{k} v_{k} \rightarrow v$ weakly in $L^{2^{*}}\left(\mathbb{R}^{N}\right)$ and $v \not \equiv 0$.

We will state now two preliminary results. The first is an easy consequence of Theorem 2.1.

Lemma 3.4 Assume that $\left\{u_{k}\right\}_{k}$ is a bounded sequence in $\mathcal{D}^{1,2}\left(\mathbb{R}^{N}\right)$ such that $u_{k} \rightarrow 0$ strongly in $L^{2^{*}}\left(\mathbb{R}^{N}\right)$. Then $u_{k} \rightarrow 0$ strongly in $L^{2_{*}}\left(\frac{1}{\left|x^{\prime}\right|^{s}}\right)$.

Proof. Recalling that

$$
2_{*}-2=\frac{2}{N-2}(2-s)
$$

and applying Hölder's inequality with exponents $\frac{N}{2-s}, \frac{N}{N-2+s}$, we obtain

$$
\begin{aligned}
\int_{\mathbb{R}^{N}} \frac{\left|u_{k}\right|^{2_{*}}}{\left|x^{\prime}\right|^{s}} d x & =\int_{\mathbb{R}^{N}} \frac{\left|u_{k}\right|^{2}}{\left|x^{\prime}\right|^{s}}\left|u_{k}\right|^{\frac{2}{N-2}(2-s)} d x \\
& \leq\left(\int_{\mathbb{R}^{N}} \frac{\left|u_{k}\right|^{\frac{2 N}{N-2+s}}}{\left|x^{\prime}\right|^{\frac{s N}{N-2+s}}} d x\right)^{\frac{N-2+s}{N}}\left(\int_{\mathbb{R}^{N}}\left|u_{k}\right|^{2^{*}} d x\right)^{\frac{2-s}{N}} .
\end{aligned}
$$

We have that

$$
\int_{\mathbb{R}^{N}}\left|u_{k}\right|^{2^{*}} d x \rightarrow 0
$$

by hypothesis. We now apply Theorem 2.1 replacing $s$ by $\frac{N s}{N-2+s}$, so that $2_{*}=2 \frac{N-s}{N-2}$ is replaced by $\frac{2 N}{N-2+s}$; notice that still $0<\frac{s N}{N-2+s}<2$. We obtain

$$
\int_{\mathbb{R}^{N}} \frac{\left|u_{k}\right|^{\frac{2 N}{N-2+s}}}{\left|x^{\prime}\right|^{\frac{s N}{N-2+s}}} d x \leq C\left(\int_{\mathbb{R}^{N}}\left|\nabla u_{n}\right|^{2}\right)^{\frac{N}{N-2+s}} \leq C,
$$

and the conclusion follows immediately.

We will also use the following version of a well known convergence result by Brézis and Lieb, [3]. 
Lemma 3.5 Assume that $\left\{w_{k}\right\}_{k} \subset \mathcal{D}^{1,2}\left(\mathbb{R}^{N}\right)$ satisfies

$$
w_{k} \rightarrow 0 \text { a.e. and } \int_{\mathbb{R}^{N}} \frac{\left|w_{k}\right|^{2 *}}{\left|x^{\prime}\right|^{s}} d x \leq C \quad \forall k .
$$

Let $\left\{\gamma_{k}\right\}_{k} \subset L^{\infty}(\mathbb{R})$ be a sequence of uniformly bounded functions, i.e., $\left\|\gamma_{k}\right\|_{\infty} \leq C$ for all $k$. Let $u \in L^{2 *}\left(\frac{1}{\left|x^{\prime}\right|^{s}}\right)$. Then we have, as $k \rightarrow \infty$,

i) $\int_{\mathbb{R}^{N}} \gamma_{k}\left(\left|x^{\prime}\right|\right) \frac{1}{\left|x^{\prime}\right|^{s}}\left|u+w_{k}\right|^{2_{*}} d x=\int_{\mathbb{R}^{N}} \gamma_{k}\left(\left|x^{\prime}\right|\right) \frac{1}{\left|x^{\prime}\right|^{s}}|u|^{2_{*}} d x$

$$
+\int_{\mathbb{R}^{N}} \gamma_{k}\left(\left|x^{\prime}\right|\right) \frac{1}{\left|x^{\prime}\right|^{s}}\left|w_{k}\right|^{2_{*}} d x+o(1) .
$$

ii) Assume also that $\gamma_{k}(x) \rightarrow 1$ a.e., as $k \rightarrow+\infty$. Then

$$
\begin{aligned}
\int_{\mathbb{R}^{N}} \gamma_{k}\left(\left|x^{\prime}\right|\right) \frac{1}{\left|x^{\prime}\right|^{s}}\left|u+w_{k}\right|^{2 *} d x= & \int_{\mathbb{R}^{N}} \frac{1}{\left|x^{\prime}\right|^{s}}|u|^{2 *} d x \\
& +\int_{\mathbb{R}^{N}} \gamma_{k}\left(\left|x^{\prime}\right|\right) \frac{1}{\left|x^{\prime}\right|^{s}}\left|w_{k}\right|^{2 *} d x+o(1) .
\end{aligned}
$$

We now begin the study of problem (2.3). Throughout this section $\left\{u_{k}\right\}_{k}$ will be a minimizing sequence for (2.3). Without loss of generality we can assume that

$$
\begin{aligned}
& \int_{\mathbb{R}^{N}} \phi\left(\left|x^{\prime}\right|\right)\left|u_{k}\right|^{2 *} d x=1, \\
& \int_{\mathbb{R}^{N}}\left|\nabla u_{k}\right|^{2} d x \rightarrow S_{\phi}, u_{k} \rightarrow u \text { weakly in } \mathcal{D}^{1,2}\left(\mathbb{R}^{N}\right) .
\end{aligned}
$$

Remark 3.6 If $u \not \equiv 0$, it is not hard to prove, for example by using a suitable version of Ekeland's variational principle, that $u$ solves the minimization problem. Therefore we could suppose in what follows that $u \equiv 0$; however we prefer to carry out all the computations in the general form to have a better insight of the compactness properties of the minimizing sequences.

In what follows we will pass several times to subsequences of $\left\{u_{k}\right\}_{k}$ and we will use the same indices. Let us define $v_{k}=u_{k}-u$, so that $\left\{v_{k}\right\}_{k}$ is a bounded sequence in $\mathcal{D}^{1,2}\left(\mathbb{R}^{N}\right)$ such that $v_{k} \rightarrow 0$ weakly in $\mathcal{D}^{1,2}\left(\mathbb{R}^{N}\right)$; passing to a subsequence we can also assume that $v_{k}(x) \rightarrow 0$ a.e., which, from now on, will be taken for granted.

From Theorem 3.3 we deduce that one of the alternatives $i$ ), ii) therein must hold. It is very easy to see that if $i$ ) holds, the sequence $\left\{u_{k}\right\}_{k}$ is actually compact in $\mathcal{D}^{1,2}$, so that in this case we obtain a solution. This is proved in the following lemma. 


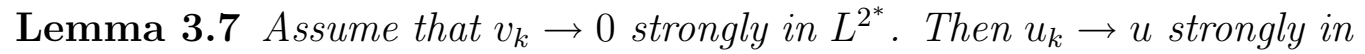
$\mathcal{D}^{1,2}\left(\mathbb{R}^{N}\right)$ and $u$ is a solution of the minimization problem (2.3).

Proof. By Lemma 3.4 we have that $v_{k} \rightarrow 0$ strongly in in $L^{2 *}\left(\frac{1}{\left|x^{\prime}\right|^{s}}\right)$, so that $u_{k} \rightarrow u$ strongly in $L^{2 *}\left(\frac{1}{\left|x^{\prime}\right|^{s}}\right)$. Then of course

$$
\begin{aligned}
\int_{\mathbb{R}^{N}} \phi\left(\left|x^{\prime}\right|\right)\left|u_{k}-u\right|^{2 *} d x & =\int_{\mathbb{R}^{N}} \phi\left(\left|x^{\prime}\right|\right)\left|x^{\prime}\right|^{s} \frac{1}{\left|x^{\prime}\right|^{s}}\left|u_{k}-u\right|^{2 *} d x \\
& \leq C \int_{\mathbb{R}^{N}} \frac{1}{\left|x^{\prime}\right|^{s}}\left|u_{k}-u\right|^{2 *} d x \rightarrow 0,
\end{aligned}
$$

which implies that

$$
\int_{\mathbb{R}^{N}} \phi\left(\left|x^{\prime}\right|\right)|u|^{2_{*}} d x=\lim _{k} \int_{\mathbb{R}^{N}} \phi\left(\left|x^{\prime}\right|\right)\left|u_{k}\right|^{2 *} d x=1 .
$$

Therefore $u \neq 0$, and

$$
J_{\phi}(u)=\int_{\mathbb{R}^{N}}|\nabla u|^{2} d x \leq \liminf _{k} \int_{\mathbb{R}^{N}}\left|\nabla u_{k}\right|^{2} d x=\liminf _{k} J\left(u_{k}\right)=S_{\phi} .
$$

This proves that $u$ minimizes $J_{\phi}$ over $\mathcal{D}^{1,2} \backslash\{0\}$. To obtain the strong convergence of $u_{k}$ in $\mathcal{D}^{1,2}$ we compute

$$
\begin{aligned}
& S_{\phi}+o(1)=\int_{\mathbb{R}^{N}}\left|\nabla u_{k}\right|^{2} d x=\int_{\mathbb{R}^{N}}\left|\nabla u_{k}-\nabla u+\nabla u\right|^{2} d x= \\
& \int_{\mathbb{R}^{N}}\left|\nabla u_{k}-\nabla u\right|^{2} d x+\int_{\mathbb{R}^{N}}|\nabla u|^{2} d x+o(1)=\int_{\mathbb{R}^{N}}\left|\nabla u_{k}-\nabla u\right|^{2} d x+S_{\phi}+o(1), \\
& \text { which gives } \int_{\mathbb{R}^{N}}\left|\nabla u_{k}-\nabla u\right|^{2} d x=o(1) .
\end{aligned}
$$

The rest of this section is devoted to the study of case $i i$ ) of Theorem 3.3. From now on we will therefore assume that $i i$ ) holds. Notice that, as $\left\{T_{k} v_{k}\right\}_{k}$ is bounded in $\mathcal{D}^{1,2}$, we can also assume, up to subsequences, that

$$
T_{k} v_{k} \rightarrow v \quad \text { weakly in } \mathcal{D}^{1,2} \text { and } T_{k} v_{k}(x) \rightarrow v(x) \text { a.e., with } v \not \equiv 0 .
$$

Let us write $T_{k} v_{k}=v+w_{k}$, so that $v_{k}=T_{k}^{-1} v+T_{k}^{-1} w_{k}$. We have therefore obtained that the minimizing sequence $u_{k}$ can be represented as

$$
u_{k}=u+T_{k}^{-1} v+T_{k}^{-1} w_{k},
$$

with the obvious properties

$$
w_{k} \rightarrow 0 \quad \text { weakly in } \mathcal{D}^{1,2} \text { and } \quad w_{k}(x) \rightarrow 0 \text { a.e. }
$$

We now begin to analyze the behavior of $J_{\phi}\left(u_{k}\right)$. The starting point is the following lemma. In its statement recall that $T_{k}=T\left(\lambda_{k}, p_{k}\right)$ and that $T_{k}^{-1}=T\left(1 / \lambda_{k},-\lambda_{k} p_{k}\right)$, for some $\lambda_{k}>0$ and $p_{k} \in \mathbb{R}^{N}$. 
Lemma 3.8 There is no subsequence of $\left(\lambda_{k}, p_{k}\right)$ converging to $\left(\lambda_{0}, p_{0}\right)$ with $\lambda_{0} \neq 0$.

Proof. We argue by contradiction by assuming that for some subsequence,

$$
\lambda_{k} \rightarrow \lambda_{0} \neq 0 \text { and } p_{k} \rightarrow p_{0} .
$$

Recall that $v_{k} \rightarrow 0$ weakly in $L^{2^{*}}$ and $T_{k} v_{k} \rightarrow v \not \equiv 0$ weakly in $L^{2^{*}}$. Fix $\varphi \in C_{0}^{\infty}\left(\mathbb{R}^{N}\right)$; then

$$
\int_{\mathbb{R}^{N}} T_{k} v_{k} \varphi d x \rightarrow \int_{\mathbb{R}^{N}} v \varphi d x
$$

On the other hand,

$$
\int_{\mathbb{R}^{N}} T_{k} v_{k} \varphi d x=\int_{\mathbb{R}^{N}} \lambda_{k}^{\frac{N-2}{2}} v_{k}\left(\lambda_{k}\left(x-p_{k}\right)\right) \varphi(x) d x=\int_{\mathbb{R}^{N}} v_{k}(y) \lambda_{k}^{-\frac{N+2}{2}} \varphi\left(\frac{y}{\lambda_{k}}+p_{k}\right) d y .
$$

From (3.6) we easily deduce that

$$
\lambda_{k}^{-\frac{N+2}{2}} \varphi\left(\frac{y}{\lambda_{k}}+p_{k}\right) \rightarrow \lambda_{0}^{-\frac{N+2}{2}} \varphi\left(\frac{y}{\lambda_{0}}+p_{0}\right) \quad \text { strongly in } L^{2^{*}}
$$

since $v_{k} \rightarrow 0$, this implies that

$$
\int_{\mathbb{R}^{N}} T_{k} v_{k} \varphi d x \rightarrow 0
$$

But $T_{k} v_{k} \rightarrow v$ weakly, so that

$$
\int_{\mathbb{R}^{N}} v \varphi d x=0
$$

This holds for all $\varphi \in C_{0}^{\infty}\left(\mathbb{R}^{N}\right)$, and of course it implies $v \equiv 0$, contradicting (3.3).

The next lemma is perhaps well known; we prove it anyway, for completeness.

Lemma 3.9 As $k \rightarrow \infty, T_{k}^{-1} v \rightarrow 0$ weakly in $L^{2^{*}}$.

Proof. Let us set $\mu_{k}=1 / \lambda_{k}$ and $q_{k}=-\lambda_{k} p_{k}$. From Lemma 3.8 we easily see that there exists a subsequence such that at least one among

$$
\mu_{k} \rightarrow 0, \quad \mu_{k} \rightarrow+\infty, \quad \text { and } \quad\left|q_{k}\right| \rightarrow+\infty
$$

is true (as usual we don't change indices, passing to subsequences). We now consider separately the three cases in (3.7) and we show that in each one we have $T_{k}^{-1} v \rightarrow 0$ weakly in $L^{2^{*}}$. 
Assume first that $\mu_{k} \rightarrow 0$ and fix $\varphi \in C_{0}^{\infty}\left(\mathbb{R}^{N}\right)$ with $\operatorname{supp} \varphi \subset B_{R}$. We obtain

$$
\begin{aligned}
& \left|\int_{\mathbb{R}^{N}} \varphi T_{k}^{-1} v d x\right|=\left|\int_{\mathbb{R}^{N}} \mu_{k}^{\frac{N-2}{2}} v\left(\mu_{k}\left(x-q_{k}\right)\right) \varphi(x) d x\right| \\
& \quad=\left|\int_{\mathbb{R}^{N}} \mu_{k}^{-\frac{N+2}{2}} v(y) \varphi\left(\frac{y}{\mu_{k}}+q_{k}\right) d y\right| \leq \int_{B_{\mu_{k} R}\left(-\mu_{k} q_{k}\right)} \mu_{k}^{-\frac{N+2}{2}}|v|\left|\varphi\left(\frac{y}{\mu_{k}}+q_{k}\right)\right| d y \\
& \leq\left(\int_{B_{\mu_{k} R}\left(-\mu_{k} q_{k}\right)}|v|^{2^{*}} d y\right)^{1 / 2^{*}}\left(\int_{B_{\mu_{k} R}\left(-\mu_{k} q_{k}\right)} \mu_{k}^{-N}\left|\varphi\left(\frac{y}{\mu_{k}}+q_{k}\right)\right|^{\frac{2 N}{N+2}} d y\right)^{\frac{N+2}{2 N}} \\
& \quad=\left(\int_{B_{\mu_{k} R}\left(-\mu_{k} q_{k}\right)}|v|^{2^{*}} d y\right)^{1 / 2^{*}}\left(\int_{B_{R}}|\varphi(y)|^{\frac{2 N}{N+2}} d y\right)^{\frac{N+2}{2 N}} .
\end{aligned}
$$

By the hypothesis $\mu_{k} \rightarrow 0$ and the absolute continuity of Lebesgue integral we have

$$
\int_{B_{\mu_{k} R}\left(-\mu_{k} q_{k}\right)}|v|^{2^{*}} d y \rightarrow 0 \quad \text { as } \quad k \rightarrow \infty
$$

so that $\int_{\mathbb{R}^{N}} \varphi T_{k}^{-1} v d x \rightarrow 0$ for all $\varphi \in C_{0}^{\infty}\left(\mathbb{R}^{N}\right)$, which of course implies $T_{k}^{-1} v \rightarrow 0$ weakly in $L^{2^{*}}$.

Let us assume now that $\mu_{k} \rightarrow+\infty$. As above we fix $\varphi \in C_{0}^{\infty}\left(\mathbb{R}^{N}\right)$, but this time we also fix $\epsilon>0$ and we choose a function $w \in C_{0}^{\infty}\left(\mathbb{R}^{N}\right)$ such that $\|v-w\|_{L^{2^{*}}}<\epsilon$. Assuming that $\operatorname{supp} \varphi \subset B_{R}$ and $\operatorname{supp} w \subset B_{R_{1}}$, we compute

$$
\begin{aligned}
& \left|\int_{\mathbb{R}^{N}} \varphi T_{k}^{-1} v d x\right| \leq\left|\int_{\mathbb{R}^{N}} \varphi\left(T_{k}^{-1} v-T_{k}^{-1} w\right) d x\right|+\left|\int_{\mathbb{R}^{N}} \varphi T_{k}^{-1} w d x\right| \\
& \leq\left\|T_{k}^{-1} v-T_{k}^{-1} w\right\|_{L^{2^{*}}}\|\varphi\|_{L^{\frac{2 N}{N+2}}}+\int_{\mathbb{R}^{N}}|\varphi|\left|T_{k}^{-1} w\right| d x \\
& \leq \epsilon\|\varphi\|_{L^{\frac{2 N}{N+2}}}+\int_{B_{R_{1} / \mu_{k}}\left(q_{k}\right)} \mu_{k}^{\frac{N-2}{2}}\left|w\left(\mu_{k}\left(x-q_{k}\right)\right)\right||\varphi| d x \\
& \leq \epsilon C+\left(\int_{B_{R_{1} / \mu_{k}}\left(q_{k}\right)} \mu_{k}^{N}\left|w\left(\mu_{k}\left(x-q_{k}\right)\right)\right|^{\frac{2 N}{N-2}} d x\right)^{\frac{N-2}{2 N}}\left(\int_{B_{R_{1} / \mu_{k}\left(q_{k}\right)}}|\varphi|^{\frac{2 N}{N+2}} d x\right)^{\frac{N+2}{2 N}} \\
& \leq \epsilon C+\left(\int_{B_{R_{1}}}|w(x)|^{\frac{2 N}{N-2}} d x\right)^{\frac{N-2}{2 N}}\left(\int_{B_{R_{1} / \mu_{k}}\left(q_{k}\right)}|\varphi|^{\frac{2 N}{N+2}} d x\right)^{\frac{N+2}{2 N}}
\end{aligned}
$$

From the hypothesis $\mu_{k} \rightarrow+\infty$ we see that $\int_{B_{R_{1} / \mu_{k}}\left(q_{k}\right)}|\varphi|^{\frac{2 N}{N+2}} d x \rightarrow 0$ (absolute continuity of Lebesgue integral). It is then straightforward to conclude that $T_{k}^{-1} v \rightarrow 0$ weakly in $L^{2^{*}}$. 
Last we assume that $\left|q_{k}\right| \rightarrow \infty$. As we have ruled out the cases $\mu_{k} \rightarrow 0$ and $\mu_{k} \rightarrow \infty$ without requiring anything on $\left\{q_{k}\right\}_{k}$, we may also assume that there exist $c_{1}, c_{2}>0$ such that $c_{1} \leq \mu_{k} \leq c_{2}$ for all $k$ (passing if necessary to a subsequence). Arguing exactly as in the first case we have, for $\varphi \in C_{0}^{\infty}\left(\mathbb{R}^{N}\right)$ with supp $\varphi \subset B_{R}$,

$$
\left|\int_{\mathbb{R}^{N}} \varphi T_{k}^{-1} v d x\right| \leq\left(\int_{B_{\mu_{k} R}\left(-\mu_{k} q_{k}\right)}|v|^{2^{*}}\right)^{\frac{1}{2^{*}}}\left(\int_{B_{R}}|\varphi|^{\frac{2 N}{N+2}} d x\right)^{\frac{N+2}{2 N}} .
$$

Since $\mu_{k} R \leq c_{2} R$ and $\left|\mu_{k} q_{k}\right| \geq c_{1}\left|q_{k}\right| \rightarrow+\infty$, we obtain

$$
\int_{B_{\mu_{k} R}\left(-\mu_{k} q_{k}\right)}|v|^{2^{*}} \rightarrow 0
$$

so that $\int_{\mathbb{R}^{N}} \varphi T_{k}^{-1} d x \rightarrow 0$ and hence $T_{k}^{-1} v \rightarrow 0$ weakly in $L^{2^{*}}$.

So in each of the three cases in (3.7) we obtain $T_{k}^{-1} v \rightarrow 0$, and the lemma is proved.

For future reference we notice that as a by-product of the previous lemma we obtain

$$
T_{k}^{-1} v \rightarrow 0 \quad \text { weakly in } \quad \mathcal{D}^{1,2} \text { and } \quad T_{k}^{-1} v(x) \rightarrow 0 \quad \text { a.e. }
$$

and also

$$
T_{k}^{-1} w_{k} \rightarrow 0 \quad \text { weakly in } \quad \mathcal{D}^{1,2} \quad \text { and } \quad T_{k}^{-1} w_{k}(x) \rightarrow 0 \quad \text { a.e., }
$$

which should be kept in mind when dealing with the representation (3.4).

We can now go on with the study of $J_{\phi}\left(u_{k}\right)$.

Lemma 3.10 There results, as $k \rightarrow+\infty$,

$$
\int_{\mathbb{R}^{N}}\left|\nabla u_{k}\right|^{2} d x=\int_{\mathbb{R}^{N}}|\nabla u|^{2} d x+\int_{\mathbb{R}^{N}}|\nabla v|^{2} d x+\int_{\mathbb{R}^{N}}\left|\nabla w_{k}\right|^{2} d x+o(1) .
$$

Proof. Since $u_{k}=u+T_{k}^{-1} v+T_{k}^{-1} w_{k}$, from (3.8) and (3.9) and simple changes of variables we obtain

$$
\begin{aligned}
\int_{\mathbb{R}^{N}}\left|\nabla u_{k}\right|^{2} d x= & \int_{\mathbb{R}^{N}}|\nabla u|^{2} d x+\int_{\mathbb{R}^{N}}\left|\nabla T_{k}^{-1} v\right|^{2} d x \\
& +\int_{\mathbb{R}^{N}}\left|\nabla T_{k}^{-1} w_{k}\right|^{2} d x+2 \int_{\mathbb{R}^{N}} \nabla u \nabla T_{k}^{-1} v d x \\
& +2 \int_{\mathbb{R}^{N}} \nabla u \nabla T_{k}^{-1} w_{k} d x+2 \int_{\mathbb{R}^{N}} \nabla T_{k}^{-1} v \nabla T_{k}^{-1} w_{k} d x \\
= & \int_{\mathbb{R}^{N}}|\nabla u|^{2} d x+\int_{\mathbb{R}^{N}}|\nabla v|^{2} d x+\int_{\mathbb{R}^{N}}\left|\nabla w_{k}\right|^{2} d x+o(1) .
\end{aligned}
$$


Remark 3.11 It is obvious that the conclusion of the preceding lemma can also be written as

$$
\int_{\mathbb{R}^{N}}\left|\nabla u_{k}\right|^{2} d x=\int_{\mathbb{R}^{N}}|\nabla u|^{2} d x+\int_{\mathbb{R}^{N}}\left|\nabla T_{1}^{-1} v\right|^{2} d x+\int_{\mathbb{R}^{N}}\left|\nabla T_{2}^{-1} w_{k}\right|^{2} d x+o(1),
$$

where $T_{1}$ and $T_{2}$ are arbitrary rescalings. We will use this freedom at the end of this section.

We now turn to the study of the term $\int_{\mathbb{R}^{N}} \phi\left|u_{k}\right|^{2 *} d x$. Most of this section is devoted to this topic.

Lemma 3.12 There results, as $k \rightarrow+\infty$,

$$
\int_{\mathbb{R}^{N}} \phi\left|u_{k}\right|^{2 *} d x=\int_{\mathbb{R}^{N}} \phi|u|^{2 *} d x+\int_{\mathbb{R}^{N}} \phi\left|T_{k}^{-1} v+T_{k}^{-1} w_{k}\right|^{2 *} d x+o(1) .
$$

Proof. The lemma follows from Lemma 3.5 writing

$$
\int_{\mathbb{R}^{N}} \phi\left(\left|x^{\prime}\right|\right)\left|u_{k}\right|^{2 *} d x=\int_{\mathbb{R}^{N}} \phi\left(\left|x^{\prime}\right|\right)\left|x^{\prime}\right|^{s} \frac{1}{\left|x^{\prime}\right|^{s}}\left|u_{k}\right|^{2 *} d x
$$

and

$$
\gamma_{k}\left(\left|x^{\prime}\right|\right)=\phi\left(\left|x^{\prime}\right|\right)\left|x^{\prime}\right|^{s} \quad \forall k
$$

We now proceed to the study of $\int_{\mathbb{R}^{N}} \phi\left|T_{k}^{-1} v+T_{k}^{-1} w_{k}\right|^{2 *} d x$, where we distinguish three cases, according to the behavior of the sequence $\left(\lambda_{k}, p_{k}\right)$. From now on we will write $p_{k}=\left(p_{k}^{\prime}, z_{k}\right) \in \mathbb{R}^{K} \times \mathbb{R}^{N-K}$. The complete analysis we need will be obtained after a series of lemmas and intermediate results.

Lemma 3.13 If $\left|p_{k}^{\prime}\right| \rightarrow+\infty$ as $k \rightarrow \infty$, then $\int_{\mathbb{R}^{N}} \frac{1}{\left|x^{\prime}\right|^{s}}\left|v\left(x+p_{k}\right)\right|^{2_{*}} d x \rightarrow 0$.

Proof. Fix $\epsilon>0$ and choose $\varphi \in C_{0}^{\infty}\left(\mathbb{R}^{N}\right)$ such that $\|v-\varphi\|<\epsilon$, with $\operatorname{supp} \varphi \subset B_{R}$; then

$$
\begin{aligned}
\int_{\mathbb{R}^{N}} & \frac{1}{\left|x^{\prime}\right|^{s}}\left|v\left(x+p_{k}\right)\right|^{2_{*}} d x=\int_{\mathbb{R}^{N}} \frac{1}{\left|x^{\prime}\right|^{s}}\left|v\left(x+p_{k}\right)-\varphi\left(x+p_{k}\right)+\varphi\left(x+p_{k}\right)\right|^{2_{*}} d x \\
& \leq C \int_{\mathbb{R}^{N}} \frac{1}{\left|x^{\prime}\right|^{s}}\left|v\left(x+p_{k}\right)-\varphi\left(x+p_{k}\right)\right|^{2_{*}} d x+C \int_{\mathbb{R}^{N}} \frac{1}{\left|x^{\prime}\right|^{s}}\left|\varphi\left(x+p_{k}\right)\right|^{2_{*}} d x \\
& \leq C \| v-\varphi||+C \int_{\mathbb{R}^{N}} \frac{1}{\left|x^{\prime}-p_{k}^{\prime}\right|^{s}}|\varphi|^{2_{*}} d x \leq C \epsilon+C \int_{B_{R}} \frac{1}{\left|x^{\prime}-p_{k}^{\prime}\right|^{s}}|\varphi|^{2_{*}} d x,
\end{aligned}
$$


where we have also used (2.2). Since $\left|x^{\prime}\right| \leq R$, we have $\left|x^{\prime}-p_{k}^{\prime}\right|^{s} \geq\left(\left|p_{k}^{\prime}\right|-R\right)^{s}$, so that

$$
\int_{B_{R}} \frac{1}{\left|x^{\prime}-p_{k}^{\prime}\right|^{s}}|\varphi|^{2 *} d x \leq \frac{1}{\left(\left|p_{k}^{\prime}\right|-R\right)^{s}} \int_{B_{R}}|\varphi|^{2 *} d x=\frac{C}{\left(\left|p_{k}^{\prime}\right|-R\right)^{s}} .
$$

As $\left|p_{k}^{\prime}\right| \rightarrow \infty$, we obtain

$$
\int_{B_{R}} \frac{1}{\left|x^{\prime}-p_{k}^{\prime}\right|^{s}}|\varphi|^{2 *} d x \rightarrow 0
$$

so that

$$
\limsup _{k} \int_{\mathbb{R}^{N}} \frac{1}{\left|x^{\prime}\right|^{s}}\left|v\left(x+p_{k}\right)\right|^{2_{*}} d x \leq C \epsilon .
$$

Since this holds for every $\epsilon>0$, we obtain the conclusion.

Lemma 3.14 Assume as before that $\left|p_{k}^{\prime}\right| \rightarrow+\infty$ as $k \rightarrow+\infty$. Then

$$
\int_{\mathbb{R}^{N}} \phi\left(\left|x^{\prime}\right|\right)\left|T_{k}^{-1} v+T_{k}^{-1} w_{k}\right|^{2 *} d x=\int_{\mathbb{R}^{N}} \phi\left(\left|x^{\prime}\right|\right)\left|T_{k}^{-1} w_{k}\right|^{2 *} d x+o(1) .
$$

Proof. Recalling the definition of $T_{k}^{-1}$, by the change of variables $x=\lambda_{k} y$ we can write

$$
\begin{aligned}
\int_{\mathbb{R}^{N}} \phi\left(\left|x^{\prime}\right|\right) \mid & T_{k}^{-1} v+\left.T_{k}^{-1} w_{k}\right|^{2 *} d x \\
& =\int_{\mathbb{R}^{N}} \phi\left(\lambda_{k}\left|y^{\prime}\right|\right) \frac{1}{\lambda_{k}^{N-s}}\left|v\left(y+p_{k}\right)+w_{k}\left(y+p_{k}\right)\right|^{2 *} \lambda_{k}^{N} d y \\
& =\int_{\mathbb{R}^{N}} \gamma_{k}\left(\left|y^{\prime}\right|\right) \frac{1}{\left|y^{\prime}\right|^{s}}\left|v\left(y+p_{k}\right)+w_{k}\left(y+p_{k}\right)\right|^{2_{*}} d y,
\end{aligned}
$$

where we have set $\gamma_{k}(r)=\phi\left(\lambda_{k} r\right) \lambda_{k}^{s} r^{s}$; notice that $\left\|\gamma_{k}\right\|_{\infty} \leq C$ uniformly in $k$.

Setting

$$
I_{k}=\left|\int_{\mathbb{R}^{N}} \gamma_{k}\left(\left|y^{\prime}\right|\right) \frac{1}{\left|y^{\prime}\right|^{s}}\left(\left|v\left(y+p_{k}\right)+w_{k}\left(y+p_{k}\right)\right|^{2 *}-\left|w_{k}\left(y+p_{k}\right)\right|^{2 *}\right) d y\right|,
$$

we want to prove that $\lim _{k} I_{k}=0$. This will end the proof. To this aim we apply the well-known inequality: $\forall p>1, \forall \epsilon>0, \exists C_{\epsilon}>0$ such that $\forall a, b \in \mathbb{R}$

$$
|| a+\left.b\right|^{p}-\left.|b|^{p}|\leq \epsilon| b\right|^{p}+C_{\epsilon}|a|^{p}
$$


to get

$$
\begin{aligned}
I_{k} & \leq C \epsilon \int_{\mathbb{R}^{N}} \frac{1}{\left|y^{\prime}\right|^{s}}\left|w_{k}\left(y+p_{k}\right)\right|^{2 *} d y+C C_{\epsilon} \int_{\mathbb{R}^{N}} \frac{1}{\left|y^{\prime}\right|^{s}}\left|v\left(y+p_{k}\right)\right|^{2 *} d y \\
& \leq C \epsilon\left\|w_{k}\right\|^{2 * / 2}+C C_{\epsilon} \int_{\mathbb{R}^{N}} \frac{1}{\left|y^{\prime}\right|^{s}}\left|v\left(y+p_{k}\right)\right|^{2_{*}} d y .
\end{aligned}
$$

By the previous lemma we have $\lim \sup _{k} I_{k} \leq C \epsilon$, so that $\lim _{k} I_{k}=0$, as we needed.

From lemmas 3.12 and 3.14 we obtain the following corollary

Corollary 3.15 If $p_{k}=\left(p_{k}^{\prime}, z_{k}\right)$ and $\left|p_{k}^{\prime}\right| \rightarrow \infty$, then

$$
\int_{\mathbb{R}^{N}} \phi\left(\left|x^{\prime}\right|\right)\left|u_{k}\right|^{2 *} d x=\int_{\mathbb{R}^{N}} \phi\left(\left|x^{\prime}\right|\right)|u|^{2 *} d x+\int_{\mathbb{R}^{N}} \phi\left(\left|x^{\prime}\right|\right)\left|T_{k}^{-1} w_{k}\right|^{2 *} d x+o(1) .
$$

Next, we study what happens in the case in which $\left\{p_{k}^{\prime}\right\}_{k}$ is bounded. Up to a subsequence, we can of course assume that

$$
p_{k}^{\prime} \rightarrow p^{\prime} \in \mathbb{R}^{K} \quad \text { as } \quad k \rightarrow \infty .
$$

Lemma 3.16 Suppose that $p_{k}=\left(p_{k}^{\prime}, z_{k}\right)$ satisfies (3.11). Then

$$
\begin{aligned}
\int_{\mathbb{R}^{N}} & \phi\left(\left|x^{\prime}\right|\right)\left|T_{k}^{-1} v+T_{k}^{-1} w_{k}\right|^{2 *} d x \\
& =\int_{\mathbb{R}^{N}} \phi\left(\left|x^{\prime}\right|\right) \frac{1}{\lambda_{k}^{N-s}}\left|v\left(\frac{x}{\lambda_{k}}+p^{\prime}\right)\right|^{2 *} d x+\int_{\mathbb{R}^{N}} \phi\left(\left|x^{\prime}\right|\right)\left|T_{k}^{-1} w_{k}\right|^{2 *} d x+o(1) .
\end{aligned}
$$

Proof. From now on, with some abuse of notation, we will denote $\left(p_{k}^{\prime}, 0\right)$ simply by $p_{k}^{\prime}$ and, likewise, $\left(0, z_{k}\right)$ by $z_{k}$.

Letting $y=\frac{x}{\lambda_{k}}+z_{k}$ we obtain

$$
\begin{aligned}
\int_{\mathbb{R}^{N}} \phi\left(\left|x^{\prime}\right|\right) & \left|T_{k}^{-1} v+T_{k}^{-1} w_{k}\right|^{2_{*}} d x \\
& =\int_{\mathbb{R}^{N}} \phi\left(\lambda_{k}\left|y^{\prime}\right|\right) \frac{1}{\lambda_{k}^{N-s}}\left|v\left(y+p_{k}^{\prime}\right)+w_{k}\left(y+p_{k}^{\prime}\right)\right|^{2 *} \lambda_{k}^{N} d y \\
& =\int_{\mathbb{R}^{N}} \gamma_{k}\left(\left|y^{\prime}\right|\right) \frac{1}{\left|y^{\prime}\right|^{s}}\left|v\left(y+p_{k}^{\prime}\right)+w_{k}\left(y+p_{k}^{\prime}\right)\right|^{2 *} d y,
\end{aligned}
$$

where we have set $\gamma_{k}(r)=\phi\left(\lambda_{k} r\right) \lambda_{k}^{s} r^{s}$ (which is uniformly bounded in $L^{\infty}$ ). 
Next we write (denoting $\left(p^{\prime}, 0\right)$ by $\left.p^{\prime}\right)$

$$
\begin{aligned}
I_{k}:= & \left|\int_{\mathbb{R}^{N}} \gamma_{k}\left(\left|y^{\prime}\right|\right) \frac{1}{\left|y^{\prime}\right|^{s}}\right| v\left(y+p_{k}^{\prime}\right)+\left.w_{k}\left(y+p_{k}^{\prime}\right)\right|^{2_{*}} d y \\
& \quad-\int_{\mathbb{R}^{N}} \gamma_{k}\left(\left|y^{\prime}\right|\right) \frac{1}{\left|y^{\prime}\right|^{s}}\left|v\left(y+p^{\prime}\right)+w_{k}\left(y+p_{k}^{\prime}\right)\right|^{2_{*}} d y \mid \\
\leq & C \int_{\mathbb{R}^{N}} \frac{1}{\left|y^{\prime}\right|^{s}}|| v\left(y+p_{k}^{\prime}\right)+\left.w_{k}\left(y+p_{k}^{\prime}\right)\right|^{2_{*}}-\left|v\left(y+p^{\prime}\right)+w_{k}\left(y+p_{k}^{\prime}\right)\right|^{2 *} \mid d y .
\end{aligned}
$$

Using again the inequality (3.10) we evaluate

$$
\begin{aligned}
&|| v\left(y+p_{k}^{\prime}\right)+\left.w_{k}\left(y+p_{k}^{\prime}\right)\right|^{2_{*}}-\left|v\left(y+p^{\prime}\right)+w_{k}\left(y+p_{k}^{\prime}\right)\right|^{2_{*}} \mid \\
&=|| v\left(y+p_{k}^{\prime}\right)-v\left(y+p^{\prime}\right)+v\left(y+p^{\prime}\right)+\left.w_{k}\left(y+p_{k}^{\prime}\right)\right|^{2_{*}} \\
& \quad-\left|v\left(y+p^{\prime}\right)+w_{k}\left(y+p_{k}^{\prime}\right)\right|^{2_{*}} \mid \\
& \leq \epsilon\left|v\left(y+p^{\prime}\right)+w_{k}\left(y+p_{k}^{\prime}\right)\right|^{2_{*}}+C_{\epsilon}\left|v\left(y+p_{k}^{\prime}\right)-v\left(y+p^{\prime}\right)\right|^{2_{*}} .
\end{aligned}
$$

Hence

$$
\begin{aligned}
I_{k} \leq C \epsilon & \int_{\mathbb{R}^{N}} \frac{1}{\left|y^{\prime}\right|^{s}}\left|v\left(y+p^{\prime}\right)+w_{k}\left(y+p_{k}^{\prime}\right)\right|^{2 *} d y \\
& \quad+C C_{\epsilon} \int_{\mathbb{R}^{N}} \frac{1}{\left|y^{\prime}\right|^{s}}\left|v\left(y+p_{k}^{\prime}\right)-v\left(y+p^{\prime}\right)\right|^{2 *} d y \\
& \leq C \epsilon+o(1),
\end{aligned}
$$

because $p_{k}^{\prime} \rightarrow p^{\prime}$. Therefore we obtain that $\lim _{k} I_{k}=0$.

From this we see, going back to (3.12), that

$$
\begin{aligned}
\int_{\mathbb{R}^{N}} \gamma_{k}\left(\left|y^{\prime}\right|\right) & \frac{1}{\left|y^{\prime}\right|^{s}}\left|v\left(y+p_{k}^{\prime}\right)+w_{k}\left(y+p_{k}^{\prime}\right)\right|^{2_{*}} d y \\
& =\int_{\mathbb{R}^{N}} \gamma_{k}\left(\left|y^{\prime}\right|\right) \frac{1}{\left|y^{\prime}\right|^{s}}\left|v\left(y+p^{\prime}\right)+w_{k}\left(y+p_{k}^{\prime}\right)\right|^{2_{*}} d y+o(1) .
\end{aligned}
$$

Notice now that we have $w_{k}\left(y+p_{k}^{\prime}\right) \rightarrow 0$ a.e. Indeed, this follows from the fact that $w_{k} \rightarrow 0$ weakly in $\mathcal{D}^{1,2}$ and $p_{k}^{\prime} \rightarrow p^{\prime}$, which imply that $w_{k}\left(y+p_{k}^{\prime}\right) \rightarrow 0$ weakly in $\mathcal{D}^{1,2}$.

We can then apply Lemma 3.5, obtaining

$$
\begin{aligned}
& \int_{\mathbb{R}^{N}} \gamma_{k}\left(\left|y^{\prime}\right|\right) \frac{1}{\left|y^{\prime}\right| s}\left|v\left(y+p^{\prime}\right)+w_{k}\left(y+p_{k}^{\prime}\right)\right|^{2 *} d y \\
& =\int_{\mathbb{R}^{N}} \gamma_{k}\left(\left|y^{\prime}\right|\right) \frac{1}{\left|y^{\prime}\right|^{s}}\left|v\left(y+p^{\prime}\right)\right|^{2_{*}} d y+\int_{\mathbb{R}^{N}} \gamma_{k}\left(\left|y^{\prime}\right|\right) \frac{1}{\left|y^{\prime}\right|^{s}}\left|w_{k}\left(y+p_{k}^{\prime}\right)\right|^{2_{*}} d y+o(1) .
\end{aligned}
$$


Now we set

$$
y=\frac{x}{\lambda_{k}}+z_{k}
$$

in the second integral of the right-hand side, and we set $y=\frac{x}{\lambda_{k}}$ in the first integral of the right-hand side. We obtain

$$
\begin{aligned}
\int_{\mathbb{R}^{N}} \phi\left(\left|x^{\prime}\right|\right)\left|T_{k}^{-1} v+T_{k}^{-1} w_{k}\right|^{2 *} d x= & \int_{\mathbb{R}^{N}} \phi\left(\left|x^{\prime}\right|\right) \frac{1}{\lambda_{k}^{N-s}}\left|v\left(\frac{x}{\lambda_{k}}+p^{\prime}\right)\right|^{2 *} d x \\
& +\int_{\mathbb{R}^{N}} \phi\left(\left|x^{\prime}\right|\right)\left|T_{k}^{-1} w_{k}\right|^{2 *} d x+o(1),
\end{aligned}
$$

and the lemma is proved.

We now refine the previous lemma, by prescribing also the behavior of $\lambda_{k}$.

Lemma 3.17 Assume that in addition to (3.11), either $\lambda_{k} \rightarrow 0$ or $\lambda_{k} \rightarrow$ $+\infty$. Then

$$
\begin{aligned}
& \int_{\mathbb{R}^{N}} \phi\left(\left|x^{\prime}\right|\right)\left|T_{k}^{-1} v+T_{k}^{-1} w_{k}\right|^{2 *} d x \leq \int_{\mathbb{R}^{N}} \frac{1}{\left|x^{\prime}\right|^{s}}\left|v\left(x+p^{\prime}\right)\right|^{2 *} d x \\
& +\int_{\mathbb{R}^{N}} \phi\left(\left|x^{\prime}\right|\right)\left|T_{k}^{-1} w_{k}\right|^{2 *} d x+o(1) .
\end{aligned}
$$

Proof. Since from the previous lemma we already have (3.13), we only have to prove that

$$
\int_{\mathbb{R}^{N}} \phi\left(\left|x^{\prime}\right|\right) \frac{1}{\lambda_{k}^{N-s}}\left|v\left(\frac{x}{\lambda_{k}}+p^{\prime}\right)\right|^{2 *} d y \leq \int_{\mathbb{R}^{N}} \frac{1}{\left|x^{\prime}\right|^{s}}\left|v\left(x+p^{\prime}\right)\right|^{2 *} d x+o(1) .
$$

To prove (3.14) we first set $x=\lambda_{k} y$ and $\gamma_{k}(r)=\phi\left(\lambda_{k} r\right)\left(\lambda_{k} r\right)^{s}$, obtaining

$$
\int_{\mathbb{R}^{N}} \phi\left(\left|x^{\prime}\right|\right) \frac{1}{\lambda_{k}^{N-s}}\left|v\left(\frac{x}{\lambda_{k}}+p^{\prime}\right)\right|^{2 *} d y=\int_{\mathbb{R}^{N}} \gamma_{k}\left(\left|y^{\prime}\right|\right) \frac{1}{\left|y^{\prime}\right|^{s}}\left|v\left(y+p^{\prime}\right)\right|^{2 *} d y .
$$

Next, as $\lambda_{k} \rightarrow 0$ or $\lambda_{k} \rightarrow+\infty$, from (3.1) we easily see that in any case we have $\lim \sup _{k} \gamma_{k}(r) \leq 1$, so that by dominated convergence we conclude

$$
\begin{aligned}
\int_{\mathbb{R}^{N}} \phi\left(\left|x^{\prime}\right|\right)\left|T_{k}^{-1} v+T_{k}^{-1} w_{k}\right|^{2 *} d x \leq & \int_{\mathbb{R}^{N}} \frac{1}{\left|x^{\prime}\right|^{s}}\left|v\left(x+p^{\prime}\right)\right|^{2 *} d x \\
& +\int_{\mathbb{R}^{N}} \phi\left(\left|x^{\prime}\right|\right)\left|T_{k}^{-1} w_{k}\right|^{2 *} d x+o(1)
\end{aligned}
$$

which completes the proof. 
From the previous lemmas we obtain the following result.

Corollary 3.18 Assume that (3.11) holds and that either $\lambda_{k} \rightarrow 0$ or $\lambda_{k} \rightarrow$ $+\infty$. Then

$$
\begin{gathered}
\int_{\mathbb{R}^{N}} \phi\left|u_{k}\right|^{2 *} d x \leq \int_{\mathbb{R}^{N}} \phi|u|^{2 *} d x+\int_{\mathbb{R}^{N}} \frac{1}{\left|x^{\prime}\right|^{s}}\left|v\left(x+p^{\prime}\right)\right|^{2 *} d x \\
+\int_{\mathbb{R}^{N}} \phi\left|T_{k}^{-1} w_{k}\right|^{2 *} d x+o(1) .
\end{gathered}
$$

In the following lemma we treat the last case in which the sequence of rescalings $\left\{T_{k}\right\}_{k}$ can induce some lack of compactness. As before we set $p_{k}=\left(p_{k}^{\prime}, z_{k}\right)$ and we denote $p_{k}^{\prime}=\left(p_{k}^{\prime}, 0\right)$ and $z_{k}=\left(0, z_{k}\right)$.

Lemma 3.19 Assume that $p_{k}^{\prime} \rightarrow p^{\prime}, \lambda_{k} \rightarrow \lambda_{0} \neq 0$ and $\left|z_{k}\right| \rightarrow+\infty$. Then

$$
\begin{aligned}
\int_{\mathbb{R}^{N}} & \phi\left(\left|x^{\prime}\right|\right)\left|T_{k}^{-1} v+T_{k}^{-1} w_{k}\right|^{2 *} d x \\
& =\int_{\mathbb{R}^{N}} \phi\left(\left|x^{\prime}\right|\right) \frac{1}{\lambda_{0}^{N-s}}\left|v\left(\frac{x}{\lambda_{0}}+p^{\prime}\right)\right|^{2 *} d x+\int_{\mathbb{R}^{N}} \phi\left(\left|x^{\prime}\right|\right)\left|T_{k}^{-1} w_{k}\right|^{2 *}+o(1) .
\end{aligned}
$$

Proof. From Lemma 3.16 we already know that

$$
\begin{aligned}
\int_{\mathbb{R}^{N}} \phi & \left(\left|x^{\prime}\right|\right)\left|T_{k}^{-1} v+T_{k}^{-1} w_{k}\right|^{2 *} d x \\
& =\int_{\mathbb{R}^{N}} \phi\left(\left|x^{\prime}\right|\right) \frac{1}{\lambda_{k}^{N-s}}\left|v\left(\frac{x}{\lambda_{k}}+p^{\prime}\right)\right|^{2 *} d x+\int_{\mathbb{R}^{N}} \phi\left(\left|x^{\prime}\right|\right)\left|T_{k}^{-1} w_{k}\right|^{2 *} d x+o(1) .
\end{aligned}
$$

To conclude we set $y=x / \lambda_{k}$ in the second integral, obtaining

$$
\int_{\mathbb{R}^{N}} \phi\left(\left|x^{\prime}\right|\right) \frac{1}{\lambda_{k}^{N-s}}\left|v\left(\frac{x}{\lambda_{k}}+p^{\prime}\right)\right|^{2 *} d x=\int_{\mathbb{R}^{N}} \phi\left(\lambda_{k}\left|y^{\prime}\right|\right) \lambda_{k}^{s}\left|y^{\prime}\right|^{s} \frac{1}{\left|y^{\prime}\right|^{s}}\left|v\left(y+p^{\prime}\right)\right|^{2 *} d y .
$$

Since

$$
\phi\left(\lambda_{k}\left|y^{\prime}\right|\right) \lambda_{k}^{s}\left|y^{\prime}\right|^{s} \rightarrow \phi\left(\lambda_{0}\left|y^{\prime}\right|\right) \lambda_{0}^{s}\left|y^{\prime}\right|^{s},
$$

by dominated convergence we immediately find that

$$
\begin{aligned}
\int_{\mathbb{R}^{N}} \phi\left(\lambda_{k}\left|y^{\prime}\right|\right) \lambda_{k}^{s}\left|y^{\prime}\right|^{s} \frac{1}{\left|y^{\prime}\right|^{s}}\left|v\left(y+p^{\prime}\right)\right|^{2 *} d y \\
=\int_{\mathbb{R}^{N}} \phi\left(\lambda_{0}\left|y^{\prime}\right|\right) \lambda_{0}^{s}\left|y^{\prime}\right|^{s} \frac{1}{\left|y^{\prime}\right|^{s}}\left|v\left(y+p^{\prime}\right)\right|^{2 *} d y+o(1) \\
=\int_{\mathbb{R}^{N}} \phi\left(\left|x^{\prime}\right|\right) \frac{1}{\lambda_{0}^{N-s}}\left|v\left(\frac{x}{\lambda_{0}}+p^{\prime}\right)\right|^{2_{*}} d y+o(1)
\end{aligned}
$$

which is what we wanted to prove. 
As above, we isolate the result we will need in a single statement.

Corollary 3.20 Assume that $p_{k}^{\prime} \rightarrow p^{\prime}, \lambda_{k} \rightarrow \lambda_{0} \neq 0$ and $\left|z_{k}\right| \rightarrow+\infty$. Then

$$
\begin{gathered}
\int_{\mathbb{R}^{N}} \phi\left|u_{k}\right|^{2_{*}} d x=\int_{\mathbb{R}^{N}} \phi|u|^{2 *} d x+\int_{\mathbb{R}^{N}} \phi \frac{1}{\lambda_{0}^{N-s}}\left|v\left(\frac{x}{\lambda_{0}}+p^{\prime}\right)\right|^{2 *} d x \\
+\int_{\mathbb{R}^{N}} \phi\left|T_{k}^{-1} w_{k}\right|^{2_{*}} d x+o(1) .
\end{gathered}
$$

Conclusion of the proof of Theorem 3.1. We now use all the results obtained so far to conclude the proof of Theorem 3.1. Before we begin we recall that we are dealing with a minimizing sequence $u_{k}$ for $J_{\phi}$ such that $\int_{\mathbb{R}^{N}} \phi\left|u_{k}\right|^{2 *} d x=1$ for all $k$. We have shown that this minimizing sequence can be represented as

$$
u_{k}=u+T_{k}^{-1} v+T_{k}^{-1} w_{k}
$$

where $T_{k}=T\left(\lambda_{k}, p_{k}\right), p_{k}=\left(p_{k}^{\prime}, z_{k}\right)$ and $v \not \equiv 0$. We also recall that

$$
\begin{aligned}
& \int_{\mathbb{R}^{N}}|\nabla u|^{2} d x \geq S\left(\int_{\mathbb{R}^{N}} \frac{1}{\left|x^{\prime}\right|^{s}}|u|^{2 *} d x\right)^{2 / 2_{*}} \quad \forall u \in \mathcal{D}^{1,2}, \\
& \int_{\mathbb{R}^{N}}|\nabla u|^{2} d x \geq S_{\phi}\left(\int_{\mathbb{R}^{N}} \phi\left(\left|x^{\prime}\right|\right)|u|^{2 *} d x\right)^{2 / 2_{*}} \quad \forall u \in \mathcal{D}^{1,2}
\end{aligned}
$$

and that our main assumption is $S_{\phi}<S$.

We will consider the three cases, depending on the behavior of $p_{k}$ and $\lambda_{k}$, which could give rise to a lack of compactness. We will show that two of them cannot hold, while the third yields the existence of a solution.

At more than one stage we will make use of the following well-known inequality: $\forall p>1 \exists C=C_{p}>0$ such that $\forall a, b \in \mathbb{R}^{+}$,

$$
(a+b)^{p} \geq a^{p}+b^{p}+C a^{p-1} b+C a b^{p-1} .
$$

A. Assume that $\left|p_{k}^{\prime}\right| \rightarrow \infty$. Then from Corollary 3.15 we have

$$
1=\int_{\mathbb{R}^{N}} \phi\left|u_{k}\right|^{2 *} d x=\int_{\mathbb{R}^{N}} \phi|u|^{2 *} d x+\int_{\mathbb{R}^{N}} \phi\left|T_{k}^{-1} w_{k}\right|^{2_{*}} d x+o(1) .
$$

From Lemma 3.10 (see also Remark 3.11) and using (3.15), we compute

$$
\begin{aligned}
& S_{\phi}^{2_{*} / 2}+o(1)=\left(\int_{\mathbb{R}^{N}}\left|\nabla u_{k}\right|^{2} d x\right)^{2_{*} / 2} \\
& \geq\left(\int_{\mathbb{R}^{N}}|\nabla u|^{2} d x\right)^{2_{*} / 2}+\left(\int_{\mathbb{R}^{N}}|\nabla v|^{2} d x\right)^{2_{*} / 2}+\left(\int_{\mathbb{R}^{N}}\left|\nabla T_{k}^{-1} w_{k}\right|^{2} d x\right)^{2_{*} / 2}+o(1) \\
& \geq S_{\phi}^{2_{*} / 2} \int_{\mathbb{R}^{N}} \phi|u|^{2_{*}} d x+S_{\phi}^{2_{*} / 2} \int_{\mathbb{R}^{N}} \phi|v|^{2_{*}} d x+S_{\phi}^{2_{*} / 2} \int_{\mathbb{R}^{N}} \phi\left|T_{k}^{-1} w_{k}\right|^{2_{*}} d x+o(1) .
\end{aligned}
$$


From (3.16) we see that $\int_{\mathbb{R}^{N}} \phi\left|T_{k}^{-1} w_{k}\right|^{2_{*}} d x=1-\int_{\mathbb{R}^{N}} \phi|u|^{2_{*}} d x+o(1)$ and hence

$$
\begin{array}{r}
S_{\phi}^{2_{*} / 2}+o(1) \geq S_{\phi}^{2_{*} / 2} \int_{\mathbb{R}^{N}} \phi|u|^{2_{*}} d x+S_{\phi}^{2_{*} / 2} \int_{\mathbb{R}^{N}} \phi|v|^{2 *} d x \\
+S_{\phi}^{2_{*} / 2}-S_{\phi}^{2_{*} / 2} \int_{\mathbb{R}^{N}} \phi|u|^{2^{*}} d x+o(1),
\end{array}
$$

that is,

$$
o(1) \geq S_{\phi}^{2 * / 2} \int_{\mathbb{R}^{N}} \phi|v|^{2 *} d x
$$

which is impossible, as $v \not \equiv 0$. Therefore this case is ruled out.

B. Assume that $p_{k}^{\prime} \rightarrow p^{\prime}$ and that either $\lambda_{k} \rightarrow 0$ or $\lambda_{k} \rightarrow \infty$. Then from Corollary 3.18 we have

$$
\begin{gathered}
1=\int_{\mathbb{R}^{N}} \phi\left|u_{k}\right|^{2 *} d x \leq \int_{\mathbb{R}^{N}} \phi|u|^{2_{*}^{*}} d x+\int_{\mathbb{R}^{N}} \frac{1}{\left|x^{\prime}\right|^{s}}\left|v\left(x+p^{\prime}\right)\right|^{2_{*}} d x \\
+\int_{\mathbb{R}^{N}} \phi\left|T_{k}^{-1} w_{k}\right|^{2_{*}} d x+o(1) .
\end{gathered}
$$

Arguing as in case $\mathbf{A}$, we obtain

$$
\begin{gathered}
S_{\phi}^{2 * / 2}+o(1) \geq S_{\phi}^{2 * / 2} \int_{\mathbb{R}^{N}} \phi|u|^{2 *} d x+S^{2 * / 2} \int_{\mathbb{R}^{N}} \frac{1}{\left|x^{\prime}\right|^{s}}\left|v\left(x+p^{\prime}\right)\right|^{2 *} d x \\
+S_{\phi}^{2 * / 2} \int_{\mathbb{R}^{N}} \phi\left|T_{k}^{-1} w_{k}\right|^{2 *} d x+o(1) .
\end{gathered}
$$

Using (3.17) this reduces to

$$
\begin{aligned}
S_{\phi}^{2_{*} / 2}+o(1) \geq & S_{\phi}^{2_{*} / 2} \int_{\mathbb{R}^{N}} \phi|u|^{2_{*}} d x+S^{2_{*} / 2} \int_{\mathbb{R}^{N}} \frac{1}{\left|x^{\prime}\right|^{s}}\left|v\left(x+p^{\prime}\right)\right|^{2_{*}} d x+S_{\phi}^{2_{*} / 2} \\
& -S_{\phi}^{2_{*} / 2} \int_{\mathbb{R}^{N}} \frac{1}{\left|x^{\prime}\right|^{s}}\left|v\left(x+p^{\prime}\right)\right|^{2_{*}} d x-S_{\phi}^{2_{*} / 2} \int_{\mathbb{R}^{N}} \phi|u|^{2_{*}} d x+o(1),
\end{aligned}
$$

that is,

$$
o(1) \geq\left(S^{2 * / 2}-S_{\phi}^{2 * / 2}\right) \int_{\mathbb{R}^{N}} \frac{1}{\left|x^{\prime}\right|^{s}}\left|v\left(x+p^{\prime}\right)\right|^{2 *} d x .
$$

This is impossible because $S>S_{\phi}$ and $v \not \equiv 0$. Also this case is ruled out.

C. At this point we are left with the last case, that is $p_{k}^{\prime} \rightarrow p^{\prime}, \lambda_{k} \rightarrow$ $\lambda_{0} \neq 0$ and $\left|z_{k}\right| \rightarrow \infty$.

Let $\hat{T}$ be the rescaling given by $\hat{T}=T\left(\lambda_{0}, p^{\prime}\right)$, so that

$$
\hat{T}^{-1} v(x)=\lambda_{0}^{-\frac{N-2}{2}} v\left(\frac{x}{\lambda_{0}}+p^{\prime}\right) .
$$


From Lemma 3.10 in the form of Remark (3.11) we can write

$$
S_{\phi}^{2_{*} / 2}+o(1)=\left(\int_{\mathbb{R}^{N}}|\nabla u|^{2} d x+\int_{\mathbb{R}^{N}}\left|\nabla \hat{T}^{-1} v\right|^{2} d x+\int_{\mathbb{R}^{N}}\left|\nabla T_{k}^{-1} w_{k}\right|^{2} d x\right)^{2 * / 2} .
$$

Applying (3.15) two times and getting rid of some extra terms we arrive at

$$
\begin{aligned}
& S_{\phi}^{2_{*} / 2}+o(1) \geq\left(\int_{\mathbb{R}^{N}}|\nabla u|^{2} d x\right)^{2_{*} / 2}+\left(\int_{\mathbb{R}^{N}}\left|\nabla \hat{T}^{-1} v\right|^{2} d x\right)^{2_{*} / 2} \\
& \quad+\left(\int_{\mathbb{R}^{N}}\left|\nabla T_{k}^{-1} w_{k}\right|^{2} d x\right)^{2_{*} / 2}+C\left(\int_{\mathbb{R}^{N}}|\nabla u|^{2} d x\right)^{2_{*} / 2-1} \int_{\mathbb{R}^{N}}\left|\nabla \hat{T}^{-1} v\right|^{2} d x \\
& \quad+C\left(\int_{\mathbb{R}^{N}}|\nabla u|^{2} d x\right)^{2 * / 2-1} \int_{\mathbb{R}^{N}}\left|\nabla T_{k}^{-1} w_{k}\right|^{2} d x \\
& \quad+C\left(\int_{\mathbb{R}^{N}}\left|\nabla \hat{T}^{-1} v\right|^{2} d x\right)^{2_{*} / 2-1} \int_{\mathbb{R}^{N}}\left|\nabla T_{k}^{-1} w_{k}\right|^{2} d x+o(1) .
\end{aligned}
$$

Now from Corollary 3.20 we have

$$
\begin{aligned}
1 & =\int_{\mathbb{R}^{N}} \phi\left|u_{k}\right|^{2 *} d x \\
& =\int_{\mathbb{R}^{N}} \phi|u|^{2_{*}} d x+\int_{\mathbb{R}^{N}} \phi\left|\hat{T}^{-1} v\right|^{2 *} d x+\int_{\mathbb{R}^{N}} \phi\left|T_{k}^{-1} w_{k}\right|_{2_{*}} d x+o(1),
\end{aligned}
$$

so that with the same estimates and substitutions we used in the previous cases we obtain

$$
\begin{aligned}
o(1) \geq & \left(\int_{\mathbb{R}^{N}}|\nabla u|^{2} d x\right)^{2 * / 2-1} \int_{\mathbb{R}^{N}}\left|\nabla \hat{T}^{-1} v\right|^{2} d x \\
& +\left(\int_{\mathbb{R}^{N}}|\nabla u|^{2} d x\right)^{2 * / 2-1} \int_{\mathbb{R}^{N}}\left|\nabla T_{k}^{-1} w_{k}\right|^{2} d x \\
& +\left(\int_{\mathbb{R}^{N}}\left|\nabla \hat{T}^{-1} v\right|^{2} d x\right)^{2_{*} / 2-1} \int_{\mathbb{R}^{N}}\left|\nabla T_{k}^{-1} w_{k}\right|^{2} d x+o(1) .
\end{aligned}
$$

As $v \not \equiv 0$, this implies that $u=0$ and that $w_{k} \rightarrow 0$ strongly in $\mathcal{D}^{1,2}$.

Since $T_{k} v_{k}=v+w_{k}$, we see that $T_{k} v_{k}-v \rightarrow 0$ strongly in $\mathcal{D}^{1,2}$, and, by invariance, $v_{k}-T_{k}^{-1} v \rightarrow 0 \quad$ strongly in $\mathcal{D}^{1,2}$.

Recalling that $u_{k}=u+v_{k}$ with $u=0$ in this case, we finally obtain that

$$
u_{k}-T_{k}^{-1} v \rightarrow 0 \quad \text { strongly in } \mathcal{D}^{1,2} \text {. }
$$

This implies, by continuity, that $\left\{T_{k}^{-1} v\right\}_{k}$ is a minimizing sequence as well. Recall now that

$$
T_{k}^{-1} v(x)=\lambda_{k}^{-\frac{N-2}{2}} v\left(\frac{x}{\lambda_{k}}+p_{k}\right)
$$


with $p_{k}=\left(p_{k}^{\prime}, z_{k}\right)$ and $\lambda_{k}$ satisfying $p_{k}^{\prime} \rightarrow p^{\prime}, \lambda_{k} \rightarrow \lambda_{0}$ and $\left|z_{k}\right| \rightarrow \infty$. As $J_{\phi}$ is invariant when its argument is shifted along the last $N-K$ coordinates, we obtain, setting $p_{k}^{\prime}=\left(p_{k}^{\prime}, 0\right)$, that also the sequence

$$
v_{k, 1}(x)=\lambda_{k}^{-\frac{N-2}{2}} v\left(\frac{x}{\lambda_{k}}+p_{k}^{\prime}\right)
$$

is minimizing for $J_{\phi}$. But it is easy to see that

$$
v_{k, 1} \rightarrow \bar{v} \quad \text { strongly in } \mathcal{D}^{1,2}
$$

where $\bar{v}(x)=\lambda_{0}^{-\frac{N-2}{2}} v\left(\frac{x}{\lambda_{0}}+p^{\prime}\right)$. Hence in this case we have constructed a minimizing sequence which converges strongly, yielding thereby a solution of the minimization problem (2.3), and Theorem 3.1 is proved.

\section{Existence results}

In this section we give some applications of Theorem 3.1. A first and easy example is the following.

Theorem 4.1 Assume that $\phi$ satisfies (2.1) and

$$
\lim _{r \rightarrow 0} \phi(r) r^{s}=\lim _{r \rightarrow \infty} \phi(r) r^{s}=1, \quad \text { with } \quad \phi(r) r^{s} \geq 1 \quad \text { for all } \quad r>0 .
$$

Then problem (2.3) admits a solution.

Proof. If $\phi(r) r^{s} \equiv 1$, then the problem reduces to (1.6), and of course $S_{\phi}=S$. This problem has been studied in [1], where the existence of a solution has been proved.

Otherwise there exists $r_{1}>0$ such that

$$
\phi\left(r_{1}\right) r_{1}^{s}>1
$$

Let $w \in \mathcal{D}^{1,2}$ be such that

$$
\int_{\mathbb{R}^{N}}|\nabla w|^{2} d x=S, \quad \text { and } \quad \int_{\mathbb{R}^{N}} \frac{1}{\left|x^{\prime}\right|^{s}}|w|^{2 *} d x=1
$$

the existence of this function is again guaranteed by [1] and we can also assume that $w>0$. Hence, by (4.1) and (4.2) we see that

$$
\int_{\mathbb{R}^{N}} \phi\left(\left|x^{\prime}\right|\right)|w|^{2 *} d x>\int_{\mathbb{R}^{N}} \frac{1}{\left|x^{\prime}\right|^{s}}|w|^{2 *} d x=1
$$


which implies that

$$
S_{\phi} \leq \frac{\int_{\mathbb{R}^{N}}|\nabla w|^{2} d x}{\left(\int_{\mathbb{R}^{N}} \phi\left(\left|x^{\prime}\right|\right)|w|^{2 *} d x\right)^{2 / 2_{*}}}<S .
$$

The conclusion follows then from Theorem 3.1.

Up to now, we have obtained existence of solutions for (2.3) when $\phi(r) r^{s} \geq 1$ (Theorem 4.1) and nonexistence of solutions when $\phi(r) r^{s} \leq 1$ and $\phi(r) r^{s} \not \equiv 1$ (Theorem 2.9). One may ask what happens when $\phi(r) r^{s}$ has some oscillations around 1 . The next theorem gives a result in this direction.

Theorem 4.2 Assume that $s N<4$ and that in addition to (2.1) and (3.1), $\phi$ satisfies also $\lim _{r \rightarrow \infty} \phi(r) r^{s}=1$ and

$$
\exists r_{0}>0, \quad \exists \beta \in(0, K-s) \quad \text { such that } \phi(r) r^{s} \geq 1+\frac{1}{r^{\beta}} \quad \text { for all } r \geq r_{0} .
$$

Then problem (2.3) has a solution.

Remark 4.3 Notice that the hypotheses of Theorem 4.2 require $\phi(r) r^{s}$ to be above 1 just for large values of $r$.

In order to prove Theorem 4.2 we need the some estimates on the decay of $w$, the solution of problem (1.6). We obtain them by an application of the following result, due to Egnell (see [11]).

Theorem 4.4 ([11]) Let $u \in \mathcal{D}^{1,2}\left(\mathbb{R}^{N}\right), u \geq 0$ be a weak solution of the equation

$$
-\Delta u=f(x, u),
$$

where $0 \leq f(x, u) \leq b(x) u^{\sigma}$ and

$$
1<\sigma<\frac{N+2}{N-2}, \quad b \in L^{\tau}\left(\mathbb{R}^{N}\right), \quad \tau=\frac{2 N}{N+2-(N-2) \sigma} .
$$

Then $\lim \sup _{|x| \rightarrow+\infty}|x|^{N-2} u(x)<+\infty$.

In the next lemma we show that the hypotheses of Theorem 4.4 are satisfied.

Lemma 4.5 Let $w$ be a solution of problem (1.6) and assume that $s N<4$. Then there exists $C>0$ such that

$$
w(x) \leq \frac{C}{|x|^{N-2}} \quad \text { as } \quad|x| \rightarrow \infty .
$$


Proof. Fix some $\sigma \in\left(1, \frac{N+2}{N-2}\right)$ very close to 1 and write the equation as $-\Delta w=b(x) w^{\sigma}$, with $b(x)=\frac{1}{\left.\left|x^{\prime}\right|\right|^{s}} w^{2_{*}-1-\sigma}$. We must show that $b \in L^{\tau}\left(\mathbb{R}^{N}\right)$, where

$$
\tau=\frac{2 N}{N+2-\sigma(N-2)}
$$

as in Theorem 4.4. To to this we apply the inequality (2.2); we just have to check that $s \tau<2$ and that the exponents match correctly, namely

$$
\frac{2(N-s \tau)}{N-2}=\left(2_{*}-1-\sigma\right) \tau .
$$

Now since $s<4 / N$ and $\tau$ is as close as we wish to $N / 2$ (by taking $\sigma$ sufficiently close to 1$)$, we see that $s \tau<2$. Checking (4.4) amounts to checking that $2 N=\left(\left(2_{*}-1-\sigma\right)(N-2)+2 s\right) \tau$ but, noticing that $\left(2_{*}-1-\sigma\right)(N-2)+2 s=N+2-\sigma(N-2)$, we immediately obtain the conclusion, by definition of $\tau$.

Before the proof of Theorem 4.2 we notice the following application of Theorem 4.4 to problem (1.3).

Corollary 4.6 Let $u$ be a solution of (1.3). Then there exists $C>0$ such that

$$
u(x) \leq \frac{C}{|x|} \quad \text { as }|x| \rightarrow \infty .
$$

Proof. Writing

$$
\phi\left(\left|x^{\prime}\right|\right) u^{3}=\phi\left(\left|x^{\prime}\right|\right)\left|x^{\prime}\right| \frac{u^{3-\sigma}}{\left|x^{\prime}\right|} u^{\sigma}=b(x) u^{\sigma}
$$

for some $\sigma \in\left(1, \frac{N+2}{N-2}\right)$, and noticing that $s N=3<4$, and that $\phi\left(\left|x^{\prime}\right|\right)\left|x^{\prime}\right| \in$ $L^{\infty}$, we can argue like in Lemma 4.5 to see that (1.3) fits in the framework of Theorem 4.4.

Proof of Theorem 4.2. In order to apply Theorem 3.1, we will prove that $S_{\phi}<S$. Let $w$ verify (4.3) and define $w_{\lambda}(x)=\lambda^{\frac{N-2}{2}} w(\lambda x)$. We compute, setting $y=\lambda x$,

$$
\begin{aligned}
\int_{\mathbb{R}^{N}} \phi\left(\left|x^{\prime}\right|\right) w_{\lambda}^{2 *}(x) d x & =\int_{\mathbb{R}^{N}} \phi\left(\left|\frac{y^{\prime}}{\lambda}\right|\right) \frac{1}{\lambda^{s}} w(y)^{2 *} d y \\
& =\int_{\mathbb{R}^{N}} \phi\left(\left|\frac{y^{\prime}}{\lambda}\right|\right) \frac{\left|y^{\prime}\right|^{s}}{\lambda^{s}} \frac{1}{\left|y^{\prime}\right|^{s}} w(y)^{2 *} d y \\
& =\int_{\mathbb{R}^{N}} \frac{1}{\left|y^{\prime}\right|^{s}} w^{2 *} d y+\int_{\mathbb{R}^{N}}\left[\phi\left(\left|\frac{y^{\prime}}{\lambda}\right|\right) \frac{\left|y^{\prime}\right|^{s}}{\lambda^{s}}-1\right] \frac{1}{\left|y^{\prime}\right|^{s}} w^{2 *} d y \\
& =1+\int_{\mathbb{R}^{N}}\left[\phi\left(\left|\frac{y^{\prime}}{\lambda}\right|\right) \frac{\left|y^{\prime}\right|^{s}}{\lambda^{s}}-1\right] \frac{1}{\left|y^{\prime}\right|^{s}} w^{2 *} d y
\end{aligned}
$$


Let us study the last integral. We write it as

$$
\begin{aligned}
\int_{\mathbb{R}^{N}}\left[\phi\left(\left|\frac{y^{\prime}}{\lambda}\right|\right) \frac{\left|y^{\prime}\right|^{s}}{\lambda^{s}}-1\right] \frac{1}{\left|y^{\prime}\right|^{s}} w^{2 *} d y & =\int_{\left|y^{\prime}\right|<\lambda r_{0}}\left[\phi\left(\left|\frac{y^{\prime}}{\lambda}\right|\right) \frac{\left|y^{\prime}\right|^{s}}{\lambda^{s}}-1\right] \frac{1}{\left|y^{\prime}\right|^{s}} w^{2_{*}} d y \\
& +\int_{\left|y^{\prime}\right| \geq \lambda r_{0}}\left[\phi\left(\left|\frac{y^{\prime}}{\lambda}\right|\right) \frac{\left|y^{\prime}\right|^{s}}{\lambda^{s}}-1\right] \frac{1}{\left|y^{\prime}\right|^{s}} w^{2^{*}} d y
\end{aligned}
$$

and we consider these two integrals separately. We have, by our assumptions,

$$
\begin{aligned}
\int_{\left|y^{\prime}\right| \geq \lambda r_{0}}\left[\phi\left(\left|\frac{y^{\prime}}{\lambda}\right|\right) \frac{\left|y^{\prime}\right|^{s}}{\lambda^{s}}-1\right] \frac{1}{\left|y^{\prime}\right|^{s}} w^{2_{*}} d y & \geq \int_{\left|y^{\prime}\right| \geq \lambda r_{0}} \frac{1}{\left|\frac{y^{\prime}}{\lambda}\right|^{\beta}} \frac{1}{\left|y^{\prime}\right|^{s}} w^{2_{*}} d y \\
& =\lambda^{\beta} \int_{\left|y^{\prime}\right| \geq \lambda r_{0}} \frac{1}{\left|y^{\prime}\right|^{s+\beta}} w^{2^{*}} d y .
\end{aligned}
$$

When $\lambda \leq 1 / r_{0}$ we obtain

$$
\lambda^{\beta} \int_{\left|y^{\prime}\right| \geq \lambda r_{0}} \frac{1}{\left|y^{\prime}\right|^{s+\beta}} w^{2_{*}} d y \geq \lambda^{\beta} \int_{\left|y^{\prime}\right| \geq 1} \frac{1}{\left|y^{\prime}\right|^{s+\beta}} w^{2_{*}} d y=a \lambda^{\beta},
$$

where $a=\int_{\left|y^{\prime}\right| \geq 1} \frac{1}{\left|y^{\prime}\right|^{s+\beta}} w^{2 *} d y$ is (positive) and finite since

$$
\int_{\left|y^{\prime}\right| \geq 1} \frac{1}{\left|y^{\prime}\right|^{s+\beta}} w^{2_{*}} d y \leq \int_{\left|y^{\prime}\right| \geq 1} \frac{1}{\left|y^{\prime}\right|^{s}} w^{2_{*}} d y \leq \int_{\mathbb{R}^{N}} \frac{1}{\left|y^{\prime}\right|^{s}} w^{2_{*}} d y=1 .
$$

Therefore we have proved that

$$
\int_{\left|y^{\prime}\right| \geq \lambda r_{0}}\left[\phi\left(\left|\frac{y^{\prime}}{\lambda}\right|\right) \frac{\left|y^{\prime}\right|^{s}}{\lambda^{s}}-1\right] \frac{1}{\left|y^{\prime}\right|^{s}} w^{2 *} d y \geq a \lambda^{\beta}
$$

with $a>0$. Concerning the other integral we obtain, writing $y=\left(y^{\prime}, z\right)$,

$$
\begin{aligned}
\left|\int_{\left|y^{\prime}\right| \leq \lambda r_{0}}\left[\phi\left(\left|\frac{y^{\prime}}{\lambda}\right|\right) \frac{\left|y^{\prime}\right|^{s}}{\lambda^{s}}-1\right] \frac{1}{\left|y^{\prime}\right|^{s}} w^{2 *} d y\right| \leq & C \int_{\left|y^{\prime}\right| \leq \lambda r_{0},|z| \leq C_{1}} \frac{1}{\left|y^{\prime}\right|^{s}} w^{2 *} d y \\
& +C \int_{\left|y^{\prime}\right| \leq \lambda r_{0},|z| \geq C_{1}} \frac{1}{\left|y^{\prime}\right|^{s}} w^{2 *} d y .
\end{aligned}
$$

As $w$ is continuous (Lemma 2.2) we first see that

$$
\begin{aligned}
\int_{\left|y^{\prime}\right| \leq \lambda r_{0},|z| \leq C_{1}} \frac{1}{\left|y^{\prime}\right|^{s}} w^{2 *} d y & \leq C \int_{\left|y^{\prime}\right| \leq \lambda r_{0},|z| \leq C_{1}} \frac{1}{\left|y^{\prime}\right|^{s}} d y \\
& \leq C \int_{0}^{\lambda r_{0}} \frac{1}{\rho^{s}} \rho^{K-1} d \rho=C \lambda^{K-s}
\end{aligned}
$$


Next, from Lemma 4.5 we obtain

$$
\begin{aligned}
& \int_{\left|y^{\prime}\right| \leq \lambda r_{0},|z| \geq C_{1}} \frac{1}{\left|y^{\prime}\right|^{s}} w^{2 *} d y \leq C \int_{\left|y^{\prime}\right| \leq \lambda r_{0},|z| \geq C_{1}} \frac{1}{\left|y^{\prime}\right|^{s}} \frac{1}{|y|^{2(N-s)}} d y \\
& \leq C \int_{\left|y^{\prime}\right| \leq \lambda r_{0},|z| \geq C_{1}} \frac{1}{\left|y^{\prime}\right|^{s}} \frac{1}{|z|^{2(N-s)}} d y^{\prime} d z=C \lambda^{K-s} \int_{|z| \geq C_{1}} \frac{1}{|z|^{2(N-s)}} d z \\
& =C \lambda^{K-s} \int_{C_{1}}^{+\infty} \frac{1}{\rho^{2(N-s)}} \rho^{N-K-1} d \rho=C \lambda^{K-s} \int_{C_{1}}^{+\infty} \frac{1}{\rho^{N+K-2 s+1}} d \rho .
\end{aligned}
$$

But since $N+K-2 s+1>1$ (because $s<K \leq N$ ), we have that

$$
\int_{C_{1}}^{+\infty} \frac{1}{\rho^{N+K-2 s+1}} d \rho<+\infty
$$

which shows that

$$
\int_{\left|y^{\prime}\right| \leq \lambda r_{0},|z| \geq C_{1}} \frac{1}{\left|y^{\prime}\right|^{s}} w^{2 *} d y \leq C \lambda^{K-s} .
$$

Collecting all the above estimates we finally arrive at

$$
\int_{\mathbb{R}^{N}} \phi\left(\left|x^{\prime}\right|\right) w_{\lambda}^{2 *} d x \geq 1+a \lambda^{\beta}-b \lambda^{K-s},
$$

with $a, b>0$. As $\beta<K-s$, when $\lambda$ is small we obtain

$$
\int_{\mathbb{R}^{N}} \phi\left(\left|x^{\prime}\right|\right) w_{\lambda}^{2 *} d x>1
$$

which implies that

$$
S_{\phi} \leq J_{\phi}\left(w_{\lambda}\right)=\frac{\int_{\mathbb{R}^{N}}\left|\nabla w_{\lambda}\right|^{2}}{\left(\int_{\mathbb{R}^{N}} \phi\left(\left|x^{\prime}\right|\right) w_{\lambda}^{2_{*}} d x\right)^{2 / 2_{*}}}<S .
$$

The conclusion follows then from Theorem 3.1.

Remark 4.7 As we said in the introduction, problem (1.1) was studied in [1]. This problem was suggested by some research in astrophysics (see [9]), where a particular form for $\phi$ was considered, namely

$$
\phi(r)=\frac{r^{2 \alpha}}{\left(1+r^{2}\right)^{\frac{1}{2}+\alpha}} \quad(\alpha>0) .
$$

For this $\phi$ the function $r \mapsto r \phi(r)$ is increasing, and so by Corollary 2.6 (recalling Remark 2.3) we have that (for $N=3$ and $p=2_{*}=4$ ) problem (1.1) has no solutions. 
Notice that not only the usual minimization problem, but the equation itself has no solutions. Anyhow, Theorem 4.2 shows that by a small perturbation of such $\phi$ we can obtain a problem which does have a solution. Indeed, let us fix $\gamma \in(2 \alpha-1,2 \alpha)$ and $\epsilon>0$, and let us define

$$
\phi_{\epsilon}(r)=\phi(r)+\epsilon \frac{r^{\gamma}}{\left(1+r^{2}\right)^{\alpha+\frac{1}{2}}} .
$$

By trivial computations one has, for large $r$ 's,

$$
\phi_{\epsilon}(r) r-1 \geq \frac{C}{r^{2 \alpha-\gamma}} .
$$

As $0<2 \alpha-\gamma<1=K-s$, the hypotheses of Theorem 4.2 are then satisfied and we obtain a solution for problem (1.3) with $\phi_{\epsilon}$ replacing $\phi$.

\section{References}

[1] Badiale, M. and Tarantello, G.: A Sobolev-Hardy inequality with applications to a nonlinear elliptic equation arising in astrophysics. Arch. Ration. Mech. Anal. 163 (2002), no. 4, 259-293.

[2] BrÉzis, H. and Kato, T.: Remarks on the Schrödinger operators with singular complex potentials. J. Math. Pures Appl. (9) 58 (1979), 137-151.

[3] BrÉzis, H. AND LiEB, E.: A relation between pointwise convergence of functionals and convergence of functionals. Proc. Amer. Math. Soc. 28 (1983), 486-490.

[4] Caffarelli, L., Kohn, R. and Nirenberg, L.: First order interpolation inequalities with weights. Compositio Math. 53 (1984), 259-275.

[5] Caldiroli, P. And Musina, R.: On the existence of extremal functions for a weighted Sobolev embedding with critical exponent. Calc. Var. Partial Differential Equations 8 (1999), no. 4, 365-387.

[6] Caldiroli, P. and Musina, R.: Stationary states for a two-dimensional singular Schrödinger equation. Boll. Unione Mat. Ital. Sez. B Artic. Ric. Mat. (8) 4 (2001), no. 3, 609-633.

[7] Caldiroli, P. And Musina, R.: Existence and non existence results for a class of nonlinear singular Sturm-Liouville equations. Adv. Differential Equations 6 (2001), 303-326.

[8] Caldiroli, P. and Musina, R.: On a class of 2-dimensional singular elliptic problems. Proc. Roy. Soc. Edinburgh Sect. A 131 (2001), no. 3, 479-497.

[9] Ciотti, L.: Dynamical models in astrophysics. Scuola Normale Superiore, Pisa 2001. 
[10] Egnell, H.: Elliptic boundary value problems with singular coefficients and critical nonlinearity. Indiana Univ. Math. J. 38 (1989), 235-251.

[11] Egnell, H.: Asymptotic results for finite energy solutions of semilinear elliptic equations. J. Differential Equations 98 (1992), 34-56.

[12] Ghoussoub, N. And Yuan, C.: Multiple solutions for quasilinear PDEs involving the critical Sobolev and Hardy exponents. Trans. Amer. Math. Soc. 352 (2000), 5703-5743.

[13] Kuzin, I. And Pohozaev, S.: Entire solutions of semilinear elliptic equations. Progress in Nonlinear Differential Equations and their Applications 33. Birkhäuser Verlag, Basel, 1997.

[14] NI, W. M.: On the equation $\Delta u+K(|x|) u^{(n+2) /(n-2)}=0$, its generalizations and applications in geometry. Indiana Univ. Math. J. 31 (1982), 439-529.

[15] Sandeep, K.: On a noncompact minimization problem of Hardy-Sobolev type. Adv. Nonlinear Stud. 2 (2002), no. 1, 81-91.

[16] Solimini, S.: A note on compactness-type properties with respect to Lorentz norms of bounded subsets of a Sobolev space. Ann. Inst. H. Poincaré Anal. Non Linéaire 12 (1995), no. 3, 319-337.

[17] Struwe, M.: Variational Methods. Springer-Verlag, Berlin Heidelberg New York, 1990.

[18] Terracini, S.: On positive entire solutions to a class of equations with a singular coefficient and critical exponent. Adv. Differential Equations 1 (1996), 241-264.

Recibido: 8 de febrero de 2002

Marino Badiale

Dipartimento di Matematica

Università di Torino

Via Carlo Alberto 10

10123 Torino, Italy

badiale@dm.unito.it

Enrico Serra

Dipartimento di Matematica

Università di Milano

Via Saldini 50

20133 Milano, Italy

serra@mat.unimi.it

This research was supported by MURST "Variational Methods and Nonlinear Differential Equations". 